TRANSACTIONS OF THE

AMERICAN MATHEMATICAL SOCIETY

Volume 358, Number 10, October 2006, Pages 4569-4603

S 0002-9947(06)03873-6

Article electronically published on May 9, 2006

\title{
THE SPECTRUM OF TWISTED DIRAC OPERATORS ON COMPACT FLAT MANIFOLDS
}

\author{
ROBERTO J. MIATELLO AND RICARDO A. PODESTÁ
}

\begin{abstract}
Let $M$ be an orientable compact flat Riemannian manifold endowed with a spin structure. In this paper we determine the spectrum of Dirac operators acting on smooth sections of twisted spinor bundles of $M$, and we derive a formula for the corresponding eta series. In the case of manifolds with holonomy group $\mathbb{Z}_{2}^{k}$, we give a very simple expression for the multiplicities of eigenvalues that allows us to compute explicitly the $\eta$-series, in terms of values of Hurwitz zeta functions, and the $\eta$-invariant. We give the dimension of the space of harmonic spinors and characterize all $\mathbb{Z}_{2}^{k}$-manifolds having asymmetric Dirac spectrum.

Furthermore, we exhibit many examples of Dirac isospectral pairs of $\mathbb{Z}_{2}^{k}$ manifolds which do not satisfy other types of isospectrality. In one of the main examples, we construct a large family of Dirac isospectral compact flat $n$-manifolds, pairwise nonhomeomorphic to each other of the order of $a^{n}$.
\end{abstract}

\section{INTRODUCTION}

The relation between the geometry of a compact Riemannian manifold $M$ and the spectral properties of the Laplace operators, $\Delta$ and $\Delta_{p}$, acting respectively on smooth functions or on smooth $p$-forms, has been studied extensively. An elliptic differential operator whose spectrum is less understood is the Dirac operator $D$. It can be defined for Riemannian manifolds having an additional structure, a spin structure. The goal of the present paper is to investigate properties of the spectrum of Dirac operators on a compact flat manifold $M$ and with a flat twist bundle. We compare it to other spectral or geometric properties of $M$.

The spectrum of the Dirac operator is explicitly known for a small class of Riemannian manifolds (see for instance $\mathrm{Ba}$, or the list in $\mathrm{AB}$, Table 1). In the context of flat manifolds, Friedrich $([\mathrm{Fr}])$ determined the spectrum of $D$ for flat tori $T_{\Lambda}=\Lambda \backslash \mathbb{R}^{n}, \Lambda$ a lattice in $\mathbb{R}^{n}$, showing the dependence on the spin structure. In [Pf] Pfäffle studied in detail the Dirac spectrum of 3-dimensional orientable compact flat manifolds, determining the eta invariants.

The goal of this paper is to study the spectrum of $D$ and the eta series for an arbitrary compact flat manifold. Such a manifold is of the form $M_{\Gamma}:=\Gamma \backslash \mathbb{R}^{n}, \Gamma$ a Bieberbach group. If $\Lambda$ denotes the translation lattice of $\Gamma$, then $F=\Lambda \backslash \Gamma$ is a finite group, the holonomy group of $M_{\Gamma}$. We note that by the Cartan-Ambrose-Singer

Received by the editors December 8, 2003 and, in revised form, May 12, 2004 and October 8, 2004.

2000 Mathematics Subject Classification. Primary 58J53; Secondary 58C22, $20 \mathrm{H} 15$.

Key words and phrases. Dirac spectrum, flat manifolds, spinors, isospectrality.

This work was supported by Conicet and Secyt-UNC.

(C)2006 American Mathematical Society Reverts to public domain 28 years from publication 
theorem any Riemannian manifold with finite holonomy group is necessarily flat. As in [Ch], we shall use the terminology $F$-manifold for a Riemannian manifold with finite holonomy group $F$. The case when $F \simeq \mathbb{Z}_{2}^{k}$ already provides a very large class of manifolds with a rich combinatorial structure (see Section 3).

The spin structures on $M_{\Gamma}$ are in one-to-one correspondence with homomorphisms $\varepsilon: \Gamma \rightarrow \operatorname{Spin}(n)$ satisfying $\mu \circ \varepsilon=r$ (see Section 1). In [MP] we have shown one cannot hear the existence of spin structures on flat manifolds. For this purpose, we obtained necessary and sufficient conditions for the existence of spin structures on $\mathbb{Z}_{2}^{k}$-manifolds. We note that Vasquez has already shown in Va that not every flat manifold admits a spin structure by giving some examples of $M$ with holonomy group $\mathbb{Z}_{2}^{k}$ having nonzero second Stiefel-Whitney class, $w_{2}(M) \neq 0$ (see also [K] and [LS]).

Every unitary complex representation $\rho: \Gamma \rightarrow \mathrm{U}(V)$ defines a flat vector bundle $E_{\rho}:=\Gamma \backslash\left(\mathbb{R}^{n} \times V\right)$ over $M_{\Gamma}$. Now, if $(L, \mathrm{~S})$ denotes the spin representation of $\operatorname{Spin}(n)$ we can consider the associated twisted spinor bundle $S_{\rho}\left(M_{\Gamma}, \varepsilon\right):=\Gamma \backslash\left(\mathbb{R}^{n} \times(\mathrm{S} \otimes V)\right)$ over $M_{\Gamma}$, where $\gamma \cdot(x, w \otimes v)=(\gamma x, L(\varepsilon(\gamma))(w) \otimes \rho(\gamma)(v))$, for $w \in \mathrm{S}, v \in V$. We denote by $D_{\rho}$ the Dirac operator acting on smooth sections of $S_{\rho}\left(M_{\Gamma}, \varepsilon\right)$. For simplicity, we shall assume throughout this paper that $\rho_{\mid \Lambda}=I d$, that is, $\rho$ induces a complex unitary representation of the holonomy group $F \simeq \Lambda \backslash \Gamma$. Similar results could be derived in more generality, for instance, assuming that $\rho_{\mid \Lambda}=\chi$, where $\chi$ is an arbitrary unitary character of $\Lambda$. However, this would make the statements of the results more complicated, without gaining too much in exchange.

We now describe the main results. In Theorem 2.5, for an arbitrary compact flat manifold $M_{\Gamma}$, we obtain formulas for the multiplicities $d_{\rho, \mu}^{ \pm}(\Gamma, \varepsilon)$ of the eigenvalues $\pm 2 \pi \mu, \mu>0$, of the Dirac operator $D_{\rho}$ associated to a spin structure $\varepsilon$, in terms of the characters $\chi_{\rho}$ of $\rho$ and $\chi_{L_{n}}, \chi_{L_{n-1} \pm}$ of the spin and half spin representations. The multiplicity formula reads as follows. If $n$ is odd, then

$$
\begin{aligned}
d_{\rho, \mu}^{ \pm}(\Gamma, \varepsilon)= & \frac{1}{|F|}\left(\sum_{\substack{\gamma \in \Lambda \backslash \Gamma \\
B \notin F_{1}}} \chi_{\rho}(\gamma) \sum_{u \in\left(\Lambda_{\varepsilon, \mu}^{*}\right)^{B}} e^{-2 \pi i u \cdot b} \chi_{L_{n-1}^{ \pm}}\left(x_{\gamma}\right)\right. \\
& \left.+\sum_{\substack{\gamma \in \Lambda \backslash \Gamma \\
B \in F_{1}}} \chi_{\rho}(\gamma) \sum_{u \in\left(\Lambda_{\varepsilon, \mu}^{*}\right)^{B}} e^{-2 \pi i u \cdot b} \chi_{\left.L_{n-1}^{ \pm \sigma(u, x}\right)}\left(x_{\gamma}\right)\right) .
\end{aligned}
$$

Here $F_{1}$ is the subset of $F$ corresponding to the elements $B L_{b} \in \Gamma$ with $n_{B}:=$ $\operatorname{dim} \operatorname{ker}(B-I d)=1$ and $\Lambda_{\varepsilon, \mu}^{*}=\left\{u \in \Lambda^{*}:\|u\|=\mu, \varepsilon(\lambda)=e^{2 \pi i \lambda \cdot u}\right.$ for every $\left.\lambda \in \Lambda\right\}$. Furthermore, for $\gamma \in \Gamma, x_{\gamma}$ is an element in the maximal torus of $\operatorname{Spin}(n-1)$ conjugate in $\operatorname{Spin}(n)$ to $\varepsilon(\gamma)$, and $\sigma\left(u, x_{\gamma}\right)$ is a sign depending on $u$ and on the conjugacy class of $x_{\gamma}$ in $\operatorname{Spin}(n-1)$.

If $n$ is even, then the formula reduces to the first summand in (0.1) with $\chi_{L_{n-1}^{ \pm}}$ replaced by $\chi_{L_{n-1}}$.

We also compute the dimension of the space of harmonic spinors (see (2.10)), showing that these can only exist for a special class of spin structures, namely, those restricting trivially to the lattice of translations $\Lambda$.

As a consequence of the theorem, we give an expression, (2.18), for the $\eta$-series $\eta_{(\Gamma, \rho, \varepsilon)}(s)$ corresponding to $D_{\rho}$ acting on sections of $S_{\rho}\left(M_{\Gamma}, \varepsilon\right)$. 
In Sections 3 and 4 , we restrict ourselves to the case of $\mathbb{Z}_{2}^{k}$-manifolds. In this case, by computing $\chi_{L_{n-1}}$ and $\chi_{L_{n-1}^{ \pm}}$we give very explicit expressions for the multiplicities. Indeed, if the spectrum is symmetric, we have that $d_{\rho, \mu}^{ \pm}(\Gamma, \varepsilon)=$ $2^{m-k-1} d_{\rho}\left|\Lambda_{\varepsilon, \mu}^{*}\right|$ for each $\mu>0$.

The spin $\mathbb{Z}_{2}^{k}$-manifolds $\left(M_{\Gamma}, \varepsilon\right)$ having asymmetric Dirac spectrum are of a very special type. This happens if and only if $n=4 r+3$ and there exists $\gamma=B L_{b} \in \Gamma$, with $n_{B}=1$ and $\chi_{\rho}(\gamma) \neq 0$, such that $B_{\mid \Lambda}=-\delta_{\varepsilon} I d$. In this case, the asymmetric spectrum is the set of eigenvalues

$$
\left\{ \pm 2 \pi \mu_{j}: \mu_{j}=\left(j+\frac{1}{2}\right)\|f\|^{-1}, j \in \mathbb{N}_{0}\right\},
$$

where $f$ satisfies $\Lambda^{B}=\mathbb{Z} f$. We furthermore have

$$
d_{\rho, \mu}^{ \pm}(\Gamma, \varepsilon)= \begin{cases}2^{m-k-1}\left(d_{\rho}\left|\Lambda_{\varepsilon, \mu}^{*}\right| \pm 2 \sigma_{\gamma}(-1)^{r+j} \chi_{\rho}(\gamma)\right), & \mu=\mu_{j} \\ 2^{m-k-1} d_{\rho}\left|\Lambda_{\varepsilon, \mu}^{*}\right|, & \mu \neq \mu_{j}\end{cases}
$$

where $\sigma_{\gamma} \in\{ \pm 1\}$. We also give an explicit expression for the eta series:

$$
\eta_{(\Gamma, \rho, \varepsilon)}(s)=(-1)^{r} \sigma_{\gamma} \chi_{\rho}(\gamma) 2^{m-k+1} \frac{\|f\|^{s}}{(4 \pi)^{s}}\left(\zeta\left(s, \frac{1}{4}\right)-\zeta\left(s, \frac{3}{4}\right)\right),
$$

where $\zeta(s, \alpha)=\sum_{j=0}^{\infty} \frac{1}{(j+\alpha)^{s}}$ denotes the generalized Hurwitz zeta function for $\alpha \in$ $(0,1]$. From this we obtain that the $\eta$-invariant of $M_{\Gamma}$ equals $\eta_{\rho}= \pm \chi_{\rho}(\gamma) 2^{[n / 2]-k}$, the sign depending on $\varepsilon$. This generalizes a result in [Pf] in the case when $n=3$. We summarize these results in Theorem 3.2 and Proposition 3.4 .

In Section 4 we compare Dirac isospectrality with other types of isospectrality (see Table 1 below); namely, isospectrality with respect to the spinor Laplacian $\Delta_{s, \rho}:=-D_{\rho}^{2}$ and to the Hodge Laplacian on $p$-forms, $\Delta_{p}$ for $0 \leq p \leq n$. We also look at the length spectrum of $M$ or $[L]$-spectrum, and the weak length spectrum of $M$ or $L$-spectrum, that is, the set of lengths of closed geodesics counted with and without multiplicities, respectively.

The information in Examples 4.3, 4.4, 4.5 is collected in Theorem 4.1. We summarize the results in the following table that shows the independence of Dirac isospectrality from other notions of isospectrality considered.

TABLE 1. Isospectrality

\begin{tabular}{|c|c|c|c|c|c|c|}
\hline$D_{\rho}$ & $\Delta_{s, \rho}$ & $\Delta_{p}(0 \leq p \leq n)$ & {$[L]$} & $L$ & Ex. & $\operatorname{dim}$ \\
\hline$Y e s$ & $Y e s$ & $N o$ (generically) & No & No & $4.3(\mathrm{i})$ & $n \geq 3$ \\
\hline$Y e s$ & $Y e s$ & $Y e s$ (if $p$ odd) & No & No & $4.3(\mathrm{iii})$ & $n=4 t$ \\
\hline No & $Y e s$ & $N o$ & $N o$ & No & $4.4(\mathrm{i})$ & $n \geq 7$ \\
\hline$Y e s / N o$ & $Y e s / N o$ & $Y e s(0 \leq p \leq n)$ & $Y e s$ & $Y e s$ & $4.5(\mathrm{i})$ & $n \geq 4$ \\
\hline$Y e s / N o$ & $Y e s / N o$ & $Y e s(0 \leq p \leq n)$ & $N o$ & $Y e s$ & $4.5(\mathrm{ii})$ & $n \geq 4$ \\
\hline
\end{tabular}


Finally, in Example 4.6, starting with Hantzsche-Wendt manifolds (see [MR]), we construct a large family (of cardinality depending exponentially on $n$ or $n^{2}$ ) of $\mathbb{Z}_{2}^{n-1}$-manifolds of dimension $2 n, n$ odd, pairwise nonhomeomorphic, and Dirac isospectral to each other.

In Section 5, by specializing our formula for $\eta(s)$, we give an expression for the eta series and eta invariant for a $p$-dimensional $\mathbb{Z}_{p}$-manifold, for each $p=4 r+3$ prime. We obtain an expression for the $\eta$-invariant involving Legendre symbols and trigonometric sums and give a list of the values for $p \leq 503$. If $p=3$, our values are in coincidence with those in $[\mathrm{Pf}]$. Our formulas for the $\eta$-series are reminiscent of those obtained in $\mathrm{HZ}$ to compute the $G$-index of elliptic operators for certain low-dimensional manifolds. In the case when $F \simeq \mathbb{Z}_{n}$ in dimension $n=4 r+3(n$ not necessarily prime), an alternative expression for the $\eta$-invariant in terms of the number of solutions of certain congruences $\bmod (n)$, has been given in [SS], with explicit calculations in the cases $n=3,7$.

\section{Preliminaries}

Bieberbach manifolds. We first review some standard facts on compact flat manifolds (see [Ch or Wo]). A Bieberbach group is a discrete, cocompact, torsion-free subgroup $\Gamma$ of the isometry group $I\left(\mathbb{R}^{n}\right)$ of $\mathbb{R}^{n}$. Such $\Gamma$ acts properly discontinuously on $\mathbb{R}^{n}$, thus $M_{\Gamma}=\Gamma \backslash \mathbb{R}^{n}$ is a compact flat Riemannian manifold with fundamental group $\Gamma$. Any such manifold arises in this way and will be referred to as a Bieberbach manifold. Any element $\gamma \in I\left(\mathbb{R}^{n}\right)=\mathrm{O}(n) \rtimes \mathbb{R}^{n}$ decomposes uniquely as $\gamma=B L_{b}$, with $B \in \mathrm{O}(n)$ and $b \in \mathbb{R}^{n}$. The translations in $\Gamma$ form a normal maximal abelian subgroup of finite index $L_{\Lambda}$, where $\Lambda$ is a lattice in $\mathbb{R}^{n}$ which is $B$-stable for each $B L_{b} \in \Gamma$. The restriction to $\Gamma$ of the canonical projection $r: \mathrm{I}\left(\mathbb{R}^{n}\right) \rightarrow \mathrm{O}(n)$ given by $B L_{b} \mapsto B$ is a homomorphism with kernel $L_{\Lambda}$, and $F:=r(\Gamma)$ is a finite subgroup of $\mathrm{O}(n)$. Thus, given a Bieberbach group $\Gamma$, there is an exact sequence $0 \rightarrow \Lambda \rightarrow \Gamma \stackrel{r}{\rightarrow} F \rightarrow 1$. The group $F \simeq \Lambda \backslash \Gamma$ is called the holonomy group of $\Gamma$ and gives the linear holonomy group of the Riemannian manifold $M_{\Gamma}$. Since we will be working with spin manifolds, we shall assume throughout this paper that $M_{\Gamma}$ is orientable, i.e. $F \subset \mathrm{SO}(n)$. The action by conjugation of $\Lambda \backslash \Gamma$ on $\Lambda$ defines an integral representation of $F$, called the holonomy representation. This representation can be rather complicated. For instance, in the case when $F \simeq \mathbb{Z}_{2}^{2}$, there are already Bieberbach groups with indecomposable holonomy representations for arbitrarily large $n$. By an $F$-manifold we understand a Riemannian manifold with holonomy group $F$. In Sections 3 and 4 of this paper we will study in detail the Dirac spectrum of $\mathbb{Z}_{2}^{k}$-manifolds.

Let $\Lambda^{*}=\left\{\lambda^{\prime} \in \mathbb{R}^{n}: \lambda \cdot \lambda^{\prime} \in \mathbb{Z}\right.$ for any $\left.\lambda \in \Lambda\right\}$ denote the dual lattice of $\Lambda$ and, for any $\mu \geq 0$, let $\Lambda_{\mu}^{*}=\left\{\lambda \in \Lambda^{*}:\|\lambda\|=\mu\right\}$. We note that this $\Lambda_{\mu}^{*}$ equals $\Lambda_{\mu^{2}}^{*}$ in the notation used in MR4, MR5], in the study of the spectrum of Laplace operators.

If $B \in \mathrm{O}(n)$ set

$$
\begin{aligned}
\left(\Lambda^{*}\right)^{B}= & \left\{\lambda \in \Lambda^{*}: B \lambda=\lambda\right\}, \quad\left(\Lambda_{\mu}^{*}\right)^{B}=\Lambda_{\mu}^{*} \cap\left(\Lambda^{*}\right)^{B}, \\
& n_{B}:=\operatorname{dim} \operatorname{ker}(B-I d)=\operatorname{dim}\left(\mathbb{R}^{n}\right)^{B} .
\end{aligned}
$$

If $\Gamma$ is a Bieberbach group, then the torsion-free condition implies that $n_{B}>0$ for any $\gamma=B L_{b} \in \Gamma$. Since $B$ preserves $\Lambda$ and $\Lambda^{*}$ we also have that $\left(\Lambda^{*}\right)^{B} \neq 0$. For 
such $\Gamma$, we set

$$
F_{1}=F_{1}(\Gamma):=\left\{B \in F=r(\Gamma): n_{B}=1\right\},
$$

where $n_{B}$ is as in (1.1).

Spin group. Let $C l(n)$ denote the Clifford algebra of $\mathbb{R}^{n}$ with respect to the standard inner product $\langle$,$\rangle on \mathbb{R}^{n}$ and let $\mathbb{C l}(n)=C l(n) \otimes \mathbb{C}$ be its complexification. If $\left\{e_{1}, \ldots, e_{n}\right\}$ is the canonical basis of $\mathbb{R}^{n}$, then a basis for $C l(n)$ is given by the set $\left\{e_{i_{1}} \ldots e_{i_{k}}: 1 \leq i_{1}<\cdots<i_{k} \leq n\right\}$. One has that $v w+w v+2\langle v, w\rangle=0$ holds for all $v, w \in \mathbb{R}^{n}$, thus $e_{i} e_{j}=-e_{j} e_{i}$ and $e_{i}{ }^{2}=-1$ for $i, j=1, \ldots, n$. Inside the group of units of $C l(n)$ we have the spin group given by

$$
\operatorname{Spin}(n)=\left\{g=v_{1} \ldots v_{k}:\left\|v_{j}\right\|=1, j=1, \ldots, k, k \text { even }\right\},
$$

which is a compact, simply connected Lie group if $n \geq 3$. There is a Lie group epimorphism with kernel $\{ \pm 1\}$ given by

$$
\mu: \operatorname{Spin}(n) \rightarrow \mathrm{SO}(n), \quad v \mapsto\left(x \rightarrow v x v^{-1}\right) .
$$

If $B_{j}$ is a matrix for $1 \leq j \leq m$, then we will abuse notation by denoting $\operatorname{diag}\left(B_{1}, \ldots, B_{m}\right)$ as the matrix having $B_{j}$ in the diagonal position $j$. Let $B(t)=$ $\left[\begin{array}{cc}\cos t-\sin t \\ \sin t & \cos t\end{array}\right]$ with $t \in \mathbb{R}$ and for $t_{1}, \ldots, t_{m} \in \mathbb{R}$ let

$$
\begin{aligned}
& x_{0}\left(t_{1}, \ldots, t_{m}\right):= \begin{cases}\operatorname{diag}\left(B\left(t_{1}\right), \ldots, B\left(t_{m}\right)\right), & \text { if } n=2 m, \\
\operatorname{diag}\left(B\left(t_{1}\right), \ldots, B\left(t_{m}\right), 1\right), & \text { if } n=2 m+1,\end{cases} \\
& x\left(t_{1}, \ldots, t_{m}\right):=\prod_{j=1}^{m}\left(\cos t_{j}+\sin t_{j} e_{2 j-1} e_{2 j}\right) \in \operatorname{Spin}(n) .
\end{aligned}
$$

Maximal tori in $\mathrm{SO}(n)$ and $\operatorname{Spin}(n)$ are respectively given by

$$
T_{0}=\left\{x_{0}\left(t_{1}, \ldots, t_{m}\right): t_{j} \in \mathbb{R}\right\}, \quad T=\left\{x\left(t_{1}, \ldots, t_{m}\right): t_{j} \in \mathbb{R}\right\} .
$$

The restriction $\mu: T \rightarrow T_{0}$ is a 2 -fold cover (see [LM]) and

$$
\mu\left(x\left(t_{1}, \ldots, t_{m}\right)\right)=x_{0}\left(2 t_{1}, \ldots, 2 t_{m}\right) .
$$

The Lie algebras of $\operatorname{Spin}(n)$ and those of $T$ are $\mathfrak{g}=\operatorname{span}\left\{e_{i} e_{j}: 1 \leq i<j \leq n\right\}$ and $\mathfrak{t}=\operatorname{span}\left\{e_{2 j-1} e_{2 j}: 1 \leq j \leq m\right\}$, respectively.

In the Appendix we have collected some specific facts on spin groups and spin representations that are used in the body of the paper.

Spin structures on flat manifolds. If $(M, g)$ is an orientable Riemannian manifold of dimension $n$, let $\mathrm{B}(M)=\bigcup_{x \in M} \mathrm{~B}_{x}(M)$ be the bundle of oriented frames on $M$ and $\pi: \mathrm{B}(M) \rightarrow M$ the canonical projection, that is, for $x \in M, \mathrm{~B}_{x}(M)$ is the set of ordered oriented orthonormal bases $\left(v_{1}, \ldots, v_{n}\right)$ of $T_{x}(M)$ and $\pi\left(\left(v_{1}, \ldots, v_{n}\right)\right)=x$. $\mathrm{B}(M)$ is a principal $\mathrm{SO}(n)$-bundle over $M$. A spin structure on $M$ is an equivariant 2-fold cover $p: \tilde{\mathrm{B}}(M) \rightarrow \mathrm{B}(M)$, where $\tilde{\pi}: \tilde{\mathrm{B}}(M) \rightarrow M$ is a principal Spin( $n$ )-bundle and $\pi \circ p=\tilde{\pi}$. A manifold in which a spin structure has been chosen is called a spin manifold.

We will be interested in spin structures on quotients $M_{\Gamma}=\Gamma \backslash \mathbb{R}^{n}$, where $\Gamma$ is a Bieberbach group. If $M=\mathbb{R}^{n}$, we have $\mathrm{B}\left(\mathbb{R}^{n}\right) \simeq \mathbb{R}^{n} \times \mathrm{SO}(n)$, thus clearly $\mathbb{R}^{n} \times \operatorname{Spin}(n)$ is a principal $\operatorname{Spin}(n)$-bundle and the map given by $I d \times \mu: \mathbb{R}^{n} \times$ $\operatorname{Spin}(n) \rightarrow \mathbb{R}^{n} \times \mathrm{SO}(n)$ is an equivariant 2 -fold cover. Thus we get a spin structure on $\mathbb{R}^{n}$ and since $\mathbb{R}^{n}$ is contractible, this is the only such structure. 
Now, if $\Gamma$ is a Bieberbach group we have a left action of $\Gamma$ on $\mathrm{B}\left(\mathbb{R}^{n}\right)$ given by $\gamma \cdot\left(x,\left(w_{1}, \ldots, w_{n}\right)\right)=\left(\gamma x,\left(\gamma_{*} w_{1}, \ldots, \gamma_{*} w_{n}\right)\right)$. If $\gamma=B L_{b}$, then $\gamma_{*} w_{j}=w_{j} B$. Fix $\left(v_{1}, \ldots, v_{n}\right) \in \mathrm{B}\left(\mathbb{R}^{n}\right)$. Since $\left(w_{1}, \ldots, w_{n}\right)=\left(v_{1} g, \ldots, v_{n} g\right)$ for some $g \in \operatorname{SO}(n)$, we see that $\gamma_{*} w_{j}=\left(v_{j} g\right) B=v_{j}(B g)$, thus the action of $\Gamma$ on $\mathrm{B}\left(\mathbb{R}^{n}\right)$ corresponds to the action of $\Gamma$ on $\mathbb{R}^{n} \times \mathrm{SO}(n)$ given by $\gamma \cdot(x, g)=(\gamma x, B g)$.

Now assume a group homomorphism is given

$$
\varepsilon: \Gamma \rightarrow \operatorname{Spin}(n) \quad \text { such that } \mu \circ \varepsilon=r,
$$

where $r(\gamma)=B$ if $\gamma=B L_{b} \in \Gamma$. In this case we can lift the left action of $\Gamma$ on $\mathrm{B}\left(\mathbb{R}^{n}\right)$ to $\tilde{\mathrm{B}}\left(\mathbb{R}^{n}\right) \simeq \mathbb{R}^{n} \times \operatorname{Spin}(n)$ via $\gamma \cdot(x, \tilde{g})=(\gamma x, \varepsilon(\gamma) \tilde{g})$. Thus we have the spin structure

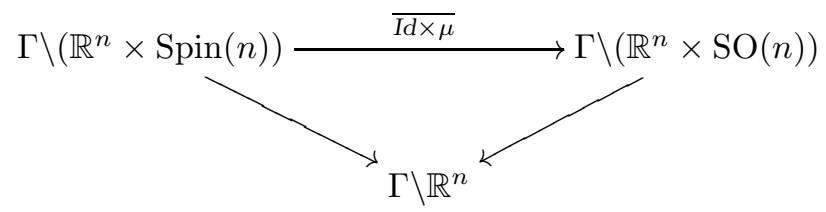

for $M_{\Gamma}$ since $\Gamma \backslash \mathrm{B}\left(\mathbb{R}^{n}\right) \simeq \mathrm{B}\left(\Gamma \backslash \mathbb{R}^{n}\right)$ and $\overline{I d \times \mu}$ is equivariant. In this way, for each such homomorphism $\varepsilon$, we obtain a spin structure on $M_{\Gamma}$. Furthermore, all spin structures on $M_{\Gamma}$ are obtained in this manner (see [Fr2, [LM]). Throughout the paper we shall denote by $\left(M_{\Gamma}, \varepsilon\right)$ a spin Bieberbach manifold endowed with the spin structure (1.7) induced by $\varepsilon$ as in (1.6).

Definition 1.1. Since $r\left(L_{\lambda}\right)=I d$ for $\lambda \in \Lambda$, then $\varepsilon(\lambda)= \pm 1$ for any $\lambda \in \Lambda$. Denote by $\delta_{\varepsilon}:=\varepsilon_{\mid \Lambda}$ the character of $\Lambda$ induced by $\varepsilon$. We will say that a spin structure $\varepsilon$ on a flat manifold $M_{\Gamma}$ is of trivial type if $\delta_{\varepsilon} \equiv 1$. For a torus $T_{\Lambda}$, the only such structure is the trivial structure corresponding to $\varepsilon \equiv 1$.

Remark 1.2. The $n$-torus admits $2^{n}$ spin structures ([Fr] $)$. An arbitrary flat manifold $M_{\Gamma}$ need not admit any. In $[\mathrm{MP}$, we give necessary and sufficient conditions for existence in the case when $\Gamma$ has holonomy group $\mathbb{Z}_{2}^{k}$ and several simple examples of flat manifolds that cannot carry a spin structure. Also, we exhibit pairs of manifolds, isospectral on $p$-forms for all $0 \leq p \leq n$, where one carries several spin (or $\operatorname{pin}^{ \pm}$) structures and the other admits none.

Twisted spinor bundles. Let $(L, S)$ be the spin representation of $\operatorname{Spin}(n)$. Then $\operatorname{dim}(\mathrm{S})=2^{m}$, with $m=\left[\frac{n}{2}\right]$. If $n$ is odd, then $L$ is irreducible, while if $n$ is even, then $\mathrm{S}=\mathrm{S}^{+} \oplus \mathrm{S}^{-}$, where $\mathrm{S}^{ \pm}$are invariant irreducible subspaces of dimension $2^{m-1}$ (see the Appendix).

The complex flat vector bundles over $M_{\Gamma}$ are in a one-to-one correspondence with complex unitary representations $\rho: \Gamma \rightarrow \mathrm{U}(V)$. For simplicity, in this paper we shall only consider representations $\rho$ of $\Gamma$ such that $\rho_{\mid \Lambda}=1$. The group $\operatorname{Spin}(n)$ acts on the right on $\tilde{\mathrm{B}}\left(\mathbb{R}^{n}\right) \times \mathrm{S} \otimes V$ by

$$
(b, w \otimes v) \cdot \tilde{g}=\left(b \tilde{g}, L\left(\tilde{g}^{-1}\right)(w) \otimes v\right),
$$

and this action defines an equivalence relation such that

$$
((x, \tilde{g}), w \otimes v) \sim((x, 1), L(\tilde{g})(w) \otimes v)
$$

for $x \in \mathbb{R}^{n}, \tilde{g} \in \operatorname{Spin}(n), w \otimes v \in \mathrm{S} \otimes V$. 
There is a bundle map from the associated bundle $\tilde{\mathrm{B}}\left(\mathbb{R}^{n}\right) \times_{L \otimes I d} \mathrm{~S} \otimes V$ onto $\mathbb{R}^{n} \times(\mathrm{S} \otimes V)$, given by $\overline{((x, \tilde{g}), w \otimes v)} \mapsto(x, L(\tilde{g}) w \otimes v)$, which is clearly an isomorphism. Given $\rho$ as above, since $\gamma \cdot \overline{((x, \tilde{g}), w \otimes v)}=\overline{((\gamma x, \varepsilon(\gamma) \tilde{g}), w \otimes \rho(\gamma) v)}$, then the corresponding action of $\gamma=B L_{b} \in \Gamma$ on $\mathbb{R}^{n} \times(\mathrm{S} \otimes V)$ is given by

$$
\gamma \cdot(x, w \otimes v)=(\gamma x, L(\varepsilon(\gamma))(w) \otimes \rho(\gamma)(v)) .
$$

In this way we get that the bundle $\Gamma \backslash\left(\tilde{\mathrm{B}}\left(\mathbb{R}^{n}\right) \times_{L \otimes I d}(\mathrm{~S} \otimes V)\right) \rightarrow \Gamma \backslash \mathbb{R}^{n}=M_{\Gamma}$, defined by $\varepsilon$, is isomorphic to

$$
S_{\rho}\left(M_{\Gamma}, \varepsilon\right):=\Gamma \backslash\left(\mathbb{R}^{n} \times(\mathrm{S} \otimes V)\right) \rightarrow \Gamma \backslash \mathbb{R}^{n}=M_{\Gamma},
$$

called the spinor bundle of $M_{\Gamma}$ with twist $V$.

Now denote by $\Gamma^{\infty}\left(S_{\rho}\left(M_{\Gamma}, \varepsilon\right)\right)$ the space of smooth sections of $S_{\rho}\left(M_{\Gamma}, \varepsilon\right)$, i.e. the space of spinor fields of $M_{\Gamma}$. Let $\psi: \mathbb{R}^{n} \rightarrow \mathbb{R}^{n} \times(\mathrm{S} \otimes V)$ be given by $\psi(x)=(x, f(x))$, where $f: \mathbb{R}^{n} \rightarrow \mathrm{S} \otimes V$ is smooth. We have that $\psi$ defines a section of $\Gamma \backslash\left(\mathbb{R}^{n} \times(\mathrm{S} \otimes V)\right)$ if and only if, for each $\gamma \in \Gamma, \psi(\gamma x) \sim \psi(x)$, that is, if and only if $\psi(\gamma x)=\tilde{\gamma} \psi(x)$, for some $\tilde{\gamma} \in \Gamma$. Since $\Gamma$ acts freely on $\mathbb{R}^{n}$, this is the case if and only if $\tilde{\gamma}=\gamma$ and furthermore $f$ satisfies $f(\gamma x)=(L \circ \varepsilon \otimes \rho)(\gamma) f(x)$. In other words, $\Gamma^{\infty}\left(S_{\rho}\left(M_{\Gamma}, \varepsilon\right)\right)$ can be identified to the space

$$
\left\{f: \mathbb{R}^{n} \rightarrow \mathrm{S} \otimes V \text { smooth }: f(\gamma x)=(L \circ \varepsilon \otimes \rho)(\gamma) f(x)\right\} .
$$

In particular, in the case of $T_{\Lambda}=\Lambda \backslash \mathbb{R}^{n}$, if $\psi(x)=(x, f(x))$ is a section of $\Lambda \backslash\left(\mathbb{R}^{n} \times(\mathrm{S} \otimes V)\right)$, then, since we have assumed that $\rho_{\mid \Lambda}=I d$, in the notation of Definition 1.1,

$$
f(x+\lambda)=(L \circ \varepsilon \otimes \rho)(\lambda) f(x)=\delta_{\varepsilon}(\lambda) f(x) .
$$

Thus $f$ is $\delta_{\varepsilon}$-equivariant. Conversely, if $f: \mathbb{R}^{n} \rightarrow \mathrm{S} \otimes V$ is $\delta_{\varepsilon}$-equivariant, then $\psi(x)=(x, f(x))$ is a spinor field on $T_{\Lambda}$.

Now $\delta_{\varepsilon} \in \operatorname{Hom}(\Lambda,\{ \pm 1\})$, hence there exists $u_{\varepsilon} \in \mathbb{R}^{n}$ such that $\delta_{\varepsilon}(\lambda)=e^{2 \pi i u_{\varepsilon} \cdot \lambda}$ for all $\lambda \in \Lambda$. Set

$$
\Lambda_{\varepsilon}^{*}:=\Lambda^{*}+u_{\varepsilon},
$$

where $\Lambda^{*}$ is the dual lattice of $\Lambda$. If $\lambda_{1}, \ldots, \lambda_{n}$ is a $\mathbb{Z}$-basis of $\Lambda$, let $\lambda_{1}^{\prime}, \ldots, \lambda_{n}^{\prime}$ be the dual basis and set

$$
J_{\varepsilon}^{ \pm}:=\left\{i \in\{1, \ldots, n\}: \varepsilon\left(L_{\lambda_{i}}\right)=\delta_{\varepsilon}\left(\lambda_{i}\right)= \pm 1\right\} .
$$

We thus have

$$
u_{\varepsilon}=\frac{1}{2} \sum_{i \in J_{\varepsilon}^{-}} \lambda_{i}^{\prime} \bmod \Lambda^{*}
$$

and furthermore

$$
\Lambda_{\varepsilon}^{*}=\bigoplus_{j \in J_{\varepsilon}^{+}} \mathbb{Z} \lambda_{j}^{\prime} \oplus \bigoplus_{j \in J_{\varepsilon}^{-}}\left(\mathbb{Z}+\frac{1}{2}\right) \lambda_{j}^{\prime}
$$

For $u \in \Lambda_{\varepsilon}^{*}, w \in \mathrm{S} \otimes V$ consider the function

$$
f_{u, w}(x):=f_{u}(x) w:=e^{2 \pi i u \cdot x} w .
$$

It is clear that $f_{u, w}$ is $\delta_{\varepsilon}$-equivariant and hence $\psi_{u, w}(x):=\left(x, f_{u, w}(x)\right)$ gives a spinor field on $T_{\Lambda}$. 


\section{The SPECTRUM OF TWISTED DiraC OPERATORS}

In this section we will introduce the Dirac operator $D_{\rho}$ acting on sections of the spinor bundle $S_{\rho}\left(M_{\Gamma}, \varepsilon\right)$ (see (1.9) ), where $(\rho, V)$ is a finite-dimensional, unitary representation of $\Gamma$. We shall denote by $\chi_{\rho}$ and $d_{\rho}$ the character and the dimension of $\rho$, respectively. In the main results of this section we will give an explicit formula for the multiplicities of the eigenvalues of twisted Dirac operators of any spin Bieberbach manifold $\left(M_{\Gamma}, \varepsilon\right)$ together with a general expression for the $\eta$-series. We shall later use this expression to compute the $\eta$-invariant for flat manifolds with holonomy group $\mathbb{Z}_{2}^{k}$ (see (3.9) ) and for certain $p$-dimensional flat manifolds with holonomy group $\mathbb{Z}_{p}, p$ prime (see (5.2)).

The spectrum of twisted Dirac operators. If $M_{\Gamma}$ is a flat manifold endowed with a spin structure $\varepsilon$, let $\Delta_{s, \rho}$ denote the twisted spinor Laplacian acting on smooth sections of the spinor bundle $S_{\rho}\left(M_{\Gamma}, \varepsilon\right)$. That is, if $\psi(x)=(x, f(x))$ is a spinor field with $f(x)=\sum_{i=1}^{d} f_{i}(x) w_{i}, f_{i}: \mathbb{R}^{n} \rightarrow \mathbb{C}$ smooth and $\left\{w_{i}: 1 \leq i \leq d\right\}$ a basis of $\mathrm{S} \otimes V\left(d=2^{m} d_{\rho}\right)$, then

$$
\Delta_{s, \rho} \psi(x)=\left(x, \sum_{i=1}^{d} \Delta f_{i}(x) w_{i}\right)
$$

where $\Delta$ is the Laplacian on functions on $M_{\Gamma}$.

It is easy to see that for $u \in \Lambda_{\varepsilon}^{*}, w \in \mathrm{S} \otimes V$, every $f_{u, w}$ as in (1.15) is an eigenfunction of $\Delta_{s, \rho}$ with eigenvalue $-4 \pi^{2}\|u\|^{2}$.

Let $\left\{e_{1}, \ldots, e_{n}\right\}$ be an orthonormal basis of $\mathbb{R}^{n}$ and let $\psi$ be as above. The twisted Dirac operator $D_{\rho}$ is defined by

$$
D_{\rho} \psi(x)=\left(x, \sum_{i=1}^{n} e_{i} \frac{\partial f}{\partial x_{i}}(x)\right)
$$

where $e_{i}$ acts by $L\left(e_{i}\right) \otimes I d$ on $\mathrm{S} \otimes V$. We will often abuse notation and assume that $D_{\rho}$ acts on the function $f$ where $\psi(x)=(x, f(x))$, writing $D_{\rho} f(x)=$ $\sum_{i=1}^{n} e_{i} \frac{\partial}{\partial x_{i}} f(x)$.

One has that $D_{\rho}$ is an elliptic, first order, essentially self-adjoint differential operator on the spinor bundle $S_{\rho}\left(M_{\Gamma}, \varepsilon\right)$ of $M_{\Gamma}$. Furthermore, $D_{\rho}$ has a discrete spectrum consisting of real eigenvalues of finite multiplicity and satisfies $D_{\rho}^{2}=$ $-\Delta_{s, \rho}$. We will denote by $\operatorname{Spec}_{D}\left(M_{\Gamma}, \varepsilon\right)$ the spectrum of $D_{\rho}$ when $\rho$ is understood.

We now compute the action of $D_{\rho}$ on $f_{u, w}$ for $u \in \Lambda_{\varepsilon}^{*}, w \in \mathrm{S} \otimes V$. We have

$$
D_{\rho} f_{u, w}(x)=\sum_{j=1}^{n} e_{j} \cdot \frac{\partial}{\partial x_{j}} e^{2 \pi i u \cdot x} w=2 \pi i e^{2 \pi i u \cdot x} u \cdot w=2 \pi i u \cdot f_{u, w}(x) .
$$

For any $u \in \mathbb{R}^{n} \backslash\{0\}$, left Clifford multiplication by $u$ on $\mathrm{S}$ is given by $u \cdot w=$ $L(u)(w)$ for $w \in \mathrm{S}$. We fix $\langle$,$\rangle an inner product on \mathrm{S}$ such that $L(u)$ is skew Hermitian for every $u \in \mathbb{R}^{n} \backslash\{0\}$, hence $\langle$,$\rangle is \operatorname{Spin}(n)$-invariant. Note that $L(u)^{2}=-\|u\|^{2} I d$. For each $u \in \Lambda_{\varepsilon}^{*}$ with $\|u\|=\mu>0$, let $\left\{w_{j}^{ \pm}\right\}_{j=1}^{2^{m-1}}$ be an orthonormal basis of the eigenspace of $L(u)$ with eigenvalue $\mp i\|u\|$. Let $\left\{v_{k}\right\}_{k=1}^{d_{\rho}}$ be an orthonormal basis of $V$. If we set

$$
f_{u, j, k}^{ \pm}(x):=f_{u, w_{j}^{ \pm}} \otimes v_{k}(x)=e^{2 \pi i u \cdot x} w_{j}^{ \pm} \otimes v_{k},
$$


for $u \in \Lambda_{\varepsilon}^{*}, 1 \leq j \leq 2^{m-1}$ and $1 \leq k \leq d_{\rho}$, then we have

$$
D_{\rho} f_{u, j, k}^{ \pm}= \pm 2 \pi\|u\| f_{u, j, k}^{ \pm}
$$

that is, $f_{u, j, k}^{ \pm} \in H_{\mu}^{ \pm}$, the space of eigensections of $D_{\rho}$ with eigenvalue $\pm 2 \pi \mu$.

If $u=0, w \neq 0$, by (1.10) the constant function $f_{0, w}(x)=w$ with $w \in \mathrm{S} \otimes V$ defines a spinor field if and only if

$$
f_{0, w}(x+\lambda)=\delta_{\varepsilon}(\lambda) f_{0, w}(x)
$$

for any $\lambda \in \Lambda$, that is, if and only if $\varepsilon=1$, the trivial spin structure. Moreover, $f_{0, w}$ is an eigenfunction of $D_{\rho}$ with eigenvalue 0, i.e. a harmonic spinor. Conversely, if $D_{\rho} f=0$, then also $\Delta_{s, \rho} f=0$, hence it follows from (2.1) that $f=f_{0, w}$, a constant function, for some $w \in \mathrm{S} \otimes V$.

We shall denote by $H_{0}$ the space of harmonic spinors. If $n$ is even, then sections of the form $f_{0, w}$ with $w \in \mathrm{S}^{+} \otimes V$ (resp. $\mathrm{S}^{-} \otimes V$ ) are often called positive (resp. negative) harmonic spinors. We have $H_{0}=H_{0}^{+} \oplus H_{0}^{-}$, where $H_{0}^{+}$and $H_{0}^{-}$ respectively denote the spaces of positive and negative harmonic spinors.

If $\mu>0$ we set $d_{\rho, \mu}^{ \pm}(\Lambda, \varepsilon):=\operatorname{dim} H_{\mu}^{ \pm}$, the multiplicities of the eigenvalues $\pm 2 \pi \mu$. If $\mu=0$, let $d_{\rho, 0}(\Lambda, \varepsilon):=\operatorname{dim} H_{0}$ and $d_{\rho, 0}^{ \pm}(\Lambda, \varepsilon):=\operatorname{dim} H_{0}^{ \pm}$, if $n$ is even.

The next result gives $\operatorname{Spec}_{D}\left(T_{\Lambda}, \varepsilon\right)$ for the torus $T_{\Lambda}$ and shows that it is determined by the cardinality of the sets

$$
\Lambda_{\varepsilon, \mu}^{*}:=\left\{v \in \Lambda_{\varepsilon}^{*}:\|v\|=\mu\right\},
$$

where $\Lambda_{\varepsilon}^{*}$ is as in (1.11).

Proposition 2.1. Let $\varepsilon$ be a spin structure on the torus $T_{\Lambda} \simeq \Lambda \backslash \mathbb{R}^{n}$ and let $m=\left[\frac{n}{2}\right]$. In the notation above, we have:

(i) $H_{0}=S \otimes V$ if $\varepsilon=1$ and $H_{0}=0$ if $\varepsilon \neq 1$.

(ii) If $\mu>0$, then

$$
H_{\mu}^{ \pm}=\operatorname{span}\left\{f_{u, j, k}^{ \pm}: u \in \Lambda_{\varepsilon, \mu}^{*}, 1 \leq j \leq 2^{m-1}, 1 \leq k \leq d_{\rho}\right\}
$$

with $f_{u, j, k}^{ \pm}$as in (2.3). The multiplicity of the eigenvalue $\pm 2 \pi \mu$ of $D_{\rho}$ thus equals

$$
d_{\rho, \mu}^{ \pm}(\Lambda, \varepsilon)=2^{m-1} d_{\rho}\left|\Lambda_{\varepsilon, \mu}^{*}\right|
$$

with $\Lambda_{\varepsilon, \mu}^{*}$ as in (2.4). Furthermore, $\left\|f_{u, j, k}^{ \pm}\right\|=\operatorname{vol}\left(T_{\Lambda}\right)^{1 / 2}$ for each $u, j, k$.

Proof. The statements in (i) are clear in light of the discussion before the proposition. Now let $L^{2}\left(\Lambda \backslash \mathbb{R}^{n} ; \delta_{\varepsilon}\right)$ denote the space

$$
\left\{f: \mathbb{R}^{n} \rightarrow \mathbb{C} \mid f(x+\lambda)=\delta_{\varepsilon}(\lambda) f(x) \text { for } \lambda \in \Lambda, x \in \mathbb{R}^{n} \text { and } \int_{T_{\Lambda}}|f|^{2}<\infty\right\}
$$

and let $L^{2}\left(\Lambda \backslash \mathbb{R}^{n}, \mathrm{~S} \otimes V ; \delta_{\varepsilon}\right)$ be defined similarly by using functions with values in $\mathrm{S} \otimes V$.

For each $u \in \Lambda_{\varepsilon}^{*}$ the function $f_{u}(x)=e^{2 \pi i u \cdot x}$ lies in $L^{2}\left(\Lambda \backslash \mathbb{R}^{n} ; \delta_{\varepsilon}\right)$. In the case $\varepsilon=1$, the Stone-Weierstrass theorem implies that the set $\left\{f_{u}: u \in \Lambda^{*}\right\}$ is a complete orthogonal system of $L^{2}\left(\Lambda \backslash \mathbb{R}^{n}\right)$. Since $\Lambda_{\varepsilon}^{*}=\Lambda^{*}+u_{\varepsilon}$, this implies that the set $\left\{f_{u}: u \in \Lambda_{\varepsilon}^{*}\right\}$ is a complete orthogonal system of $L^{2}\left(\Lambda \backslash \mathbb{R}^{n} ; \delta_{\varepsilon}\right)$. Now, if for each given $u \in \Lambda_{\varepsilon}^{*}$, we choose an orthonormal basis $\mathcal{B}_{u}$ of $\mathrm{S} \otimes V$ of eigenvectors of $L(u) \otimes I d$, then this clearly implies that the set

$$
\left\{f_{u, w}(x): u \in \Lambda_{\varepsilon}^{*}, w \in \mathcal{B}_{u}\right\}
$$


is a complete orthogonal system of $L^{2}\left(\Lambda \backslash \mathbb{R}^{n}, \mathrm{~S} \otimes V ; \delta_{\varepsilon}\right)$. Furthermore each $f_{u, w}$ is an eigenfunction of $D_{\rho}$ with eigenvalue $\pm 2 \pi\|u\|$, therefore (ii) follows.

Remark 2.2. If $\Lambda$ is the canonical (or cubic) lattice, then

$$
\left|\Lambda_{\varepsilon, \mu}^{*}\right|=\left|\left\{\left(m_{1}, \ldots, m_{n}\right) \in \mathbb{Z}^{n}: \sum_{j \in J_{\varepsilon}^{+}} m_{j}^{2}+\sum_{j \in J_{\varepsilon}^{-}}\left(m_{j}+\frac{1}{2}\right)^{2}=\mu^{2}\right\}\right|,
$$

where $J_{\varepsilon}^{ \pm}$(see (1.12) ) are computed with respect to the canonical basis of $\mathbb{R}^{n}$. We observe that $\left|J_{\varepsilon}^{-}\right|$(or $\left|J_{\varepsilon}^{+}\right|$, see (1.14) ) determines the multiplicity of the eigenvalue $\pm 2 \pi \mu$, for any $\mu>0$, by (2.6) and by the multiplicity formula in (ii) of Proposition 2.1. The converse also holds, thus we have that $\operatorname{Spec}_{D}\left(T_{\Lambda}, \varepsilon\right)=\operatorname{Spec}_{D}\left(T_{\Lambda}, \varepsilon^{\prime}\right)$ if and only if $\left|J_{\varepsilon}^{-}\right|=\left|J_{\varepsilon^{\prime}}^{-}\right|$.

Our next goal is to obtain a formula for the multiplicities of the eigenvalues $\pm 2 \pi \mu, \mu \geq 0$, of the Dirac operator acting on twisted spinor fields of an arbitrary spin flat manifold $\left(M_{\Gamma}, \varepsilon\right)$. We shall see that only the elements in $\Gamma$ such that $B \in F_{1}$ (see (1.2)) will contribute to the formula. One of the key ingredients in the formula will be a sign, attached to each pair $\gamma, u$, with $\gamma=B L_{b}, u \in \Lambda^{*}$ fixed by $B$. This sign appears when comparing the conjugacy classes of two elements $x, y$ in $\operatorname{Spin}(n-1)$ that are conjugate in $\operatorname{Spin}(n)$. By Lemma 6.2 given two such elements $x, y$, then either $y$ is conjugate to $x$ or to $-e_{1} x e_{1}$ in $\operatorname{Spin}(n-1)$ (see the Appendix). In what follows we shall write $x \sim y$ if $x, y$ are conjugate in $\operatorname{Spin}(n-1)$.

If $\gamma=B L_{b} \in \Lambda \backslash \Gamma$ and $u \in\left(\Lambda_{\varepsilon}^{*}\right)^{B} \backslash\{0\}$, let $h_{u} \in \operatorname{Spin}(n)$ be such that $h_{u} u h_{u}^{-1}=$ $\|u\| e_{n}$. Therefore, since $B u=u, h_{u} \varepsilon(\gamma) h_{u}^{-1} \in \operatorname{Spin}(n-1)$, by the comments after Definition 6.5.

We shall make use of the following definitions.

Definition 2.3. Fix an element $x_{\gamma}$ in the maximal torus $T$ of $\operatorname{Spin}(n-1)$ (see (1.5)) that is conjugate in $\operatorname{Spin}(n)$ to $\varepsilon(\gamma)$. Define $\sigma_{\varepsilon}\left(u, x_{\gamma}\right)=1$ if $h_{u} \varepsilon(\gamma) h_{u}^{-1}$ is conjugate to $x_{\gamma}$ in $\operatorname{Spin}(n-1)$, and $\sigma_{\varepsilon}\left(u, x_{\gamma}\right)=-1$ otherwise (in this case $\left.h_{u} \varepsilon(\gamma) h_{u}^{-1} \sim-e_{1} x_{\gamma} e_{1}\right)$.

Note that $\sigma_{\varepsilon}\left(u, x_{\gamma}\right)$ is independent of the choice of $h_{u}$. For simplicity, we shall simply write $\sigma\left(u, x_{\gamma}\right)$ when $\varepsilon$ is understood.

Remark 2.4. If $n$ is even, then $\sigma\left(u, x_{\gamma}\right)=1$ for all $u$, by Lemma 6.2. Also, if $\gamma$ is such that $n_{B}>1$, then, by arguing as at the end of the proof of Lemma 6.2. we see that $\sigma\left(u, x_{\gamma}\right)=1$ for all $u$. Furthermore, if $x_{\gamma}$ is not conjugate to $-e_{1} x_{\gamma} e_{1}$ in $\operatorname{Spin}(n)$, then, by the definition, $\sigma\left(u,-e_{1} x_{\gamma} e_{1}\right)=-\sigma\left(u, x_{\gamma}\right)$. Moreover $\sigma\left(-u, x_{\gamma}\right)=-\sigma\left(u, x_{\gamma}\right)$ and $\sigma\left(\alpha u, x_{\gamma}\right)=\sigma\left(u, x_{\gamma}\right)$ for any $\alpha>0$, since we may take $h_{-u}=e_{1} h_{u}$ and $h_{\alpha u}=h_{u}$, respectively.

Now, let as usual $\chi_{\rho}, \chi_{L}$ and $\chi_{L^{ \pm}}$denote the characters of $\rho, L$ and $L^{ \pm}$, respectively. Keeping the notation above, for each $\gamma=B L_{b} \in \Gamma, \mu>0$, we set

$$
e_{\mu, \gamma, \sigma}\left(\delta_{\varepsilon}\right):=\sum_{u \in\left(\Lambda_{\varepsilon, \mu}^{*}\right)^{B}} \sigma\left(u, x_{\gamma}\right) e^{-2 \pi i u \cdot b},
$$

where $\left(\Lambda_{\varepsilon, \mu}^{*}\right)^{B}=\left\{v \in \Lambda_{\varepsilon, \mu}^{*}: B v=v\right\}$. When $\sigma=1$ we just write $e_{\mu, \gamma}\left(\delta_{\varepsilon}\right)$ for $e_{\mu, \gamma, 1}\left(\delta_{\varepsilon}\right)$.

We are now in a position to prove the main result in this section. 
Theorem 2.5. Let $\Gamma$ be a Bieberbach group with translation lattice $\Lambda$ and holonomy group $F \simeq \Lambda \backslash \Gamma$. Assume $M_{\Gamma}=\Gamma \backslash \mathbb{R}^{n}$ is a spin compact flat manifold, with spin structure $\varepsilon$. Then, if $n$ is even, for each $\mu>0$ the multiplicity of the eigenvalue $\pm 2 \pi \mu$ of $D_{\rho}$ is given by

$$
d_{\rho, \mu}^{ \pm}(\Gamma, \varepsilon)=\frac{1}{|F|} \sum_{\gamma \in \Lambda \backslash \Gamma} \chi_{\rho}(\gamma) e_{\mu, \gamma}\left(\delta_{\varepsilon}\right) \chi_{L_{n-1}}\left(x_{\gamma}\right) .
$$

If $n$ is odd, then

$$
\begin{aligned}
d_{\rho, \mu}^{ \pm}(\Gamma, \varepsilon)= & \frac{1}{|F|}\left(\sum_{\substack{\gamma \in \Lambda \backslash \Gamma \\
B \notin F_{1}}} \chi_{\rho}(\gamma) e_{\mu, \gamma}\left(\delta_{\varepsilon}\right) \chi_{L_{n-1}^{ \pm}}\left(x_{\gamma}\right)\right. \\
& \left.+\sum_{\substack{\gamma \in \Lambda \backslash \Gamma \\
B \in F_{1}}} \chi_{\rho}(\gamma) \sum_{u \in\left(\Lambda_{\varepsilon, \mu}^{*}\right)^{B}} e^{-2 \pi i u \cdot b} \chi_{\left.L_{n-1}^{ \pm \sigma(u, x} \gamma\right)}\left(x_{\gamma}\right)\right)
\end{aligned}
$$

with $e_{\mu, \gamma}\left(\delta_{\varepsilon}\right)$ as in (2.7), $F_{1}$ as in (1.2), and $x_{\gamma}, \sigma\left(u, x_{\gamma}\right)$ as in Definition 2.3,

Let $\mu=0$. If $\varepsilon_{\mid \Lambda} \neq 1$, then $d_{\rho, 0}(\Gamma, \varepsilon)=0$. If $\varepsilon_{\mid \Lambda}=1$, then

$$
d_{\rho, 0}(\Gamma, \varepsilon)=\frac{1}{|F|} \sum_{\gamma \in \Lambda \backslash \Gamma} \chi_{\rho}(\gamma) \chi_{L_{n}}(\varepsilon(\gamma))=\operatorname{dim}(S \otimes V)^{F} .
$$

We note that the summands in (2.8) and in (2.9) are independent of the representative $\gamma \bmod \Lambda$ and of the choice of $x_{\gamma}$, but in general the individual factors are not.

Proof. We proceed initially as in [MR3], [MR4]. We have that

$$
L^{2}\left(S_{\rho}\left(M_{\Gamma}, \varepsilon\right)\right) \simeq L^{2}\left(S_{\rho}\left(T_{\Lambda}, \varepsilon\right)\right)^{\Gamma}=\bigoplus_{\mu>0}\left(\left(H_{\mu}^{+}\right)^{\Gamma} \oplus\left(H_{\mu}^{-}\right)^{\Gamma}\right) \oplus H_{0}{ }^{\Gamma} .
$$

Thus, $d_{\rho, \mu}^{ \pm}(\Gamma, \varepsilon)=\operatorname{dim}\left(H_{\mu}^{ \pm}\right)^{\Gamma}$, for $\mu>0$, and $d_{\rho, 0}(\Gamma, \varepsilon)=\operatorname{dim} H_{0}{ }^{\Gamma}$. One has a projection $p_{\mu}^{ \pm}$from $H_{\mu}^{ \pm}$onto $\left(H_{\mu}^{ \pm}\right)^{\Gamma}$ :

$$
p_{\mu}^{ \pm}=\frac{1}{|\Lambda \backslash \Gamma|} \sum_{\gamma \in \Lambda \backslash \Gamma} \gamma_{\mid H_{\mu}^{ \pm}},
$$

hence

$$
\operatorname{dim}\left(H_{\mu}^{ \pm}\right)^{\Gamma}=\operatorname{tr} p_{\mu}^{ \pm}=\frac{1}{|\Lambda \backslash \Gamma|} \sum_{\gamma \in \Lambda \backslash \Gamma} \operatorname{tr}\left(\gamma_{\mid H_{\mu}^{ \pm}}\right)
$$

and similarly for $\operatorname{dim} H_{0}{ }^{\Gamma}$, with $H_{0}$ in place of $H_{\mu}^{ \pm}$.

Thus, by (ii) of Proposition 2.1 we have to compute, for $\gamma \in \Gamma$,

$$
\operatorname{tr} \gamma_{\mid H_{\mu}^{ \pm}}=\frac{1}{\operatorname{vol}\left(T_{\Lambda}\right)} \sum_{u \in \Lambda_{\varepsilon, \mu}^{*}} \sum_{j=1}^{2^{m-1}} \sum_{k=1}^{d_{\rho}}\left\langle\gamma \cdot f_{u, j, k}^{ \pm}, f_{u, j, k}^{ \pm}\right\rangle .
$$

Recall, from (1.8), that $\gamma \in \Gamma$ acts by $\gamma \cdot \psi(x)=(\gamma x,(L \circ \varepsilon \otimes \rho)(\gamma) f(x))$ on $\psi(x)=(x, f(x))$. Thus there is an action of $\gamma$ on $f$ given by

$$
\gamma \cdot f(x)=(L \circ \varepsilon \otimes \rho)(\gamma) f\left(\gamma^{-1} x\right) .
$$

Since $\gamma^{-1}=L_{-b} B^{-1}$ by (2.3) we have

$$
\begin{aligned}
\gamma \cdot f_{u, j, k}^{ \pm}(x) & =(L \circ \varepsilon \otimes \rho)(\gamma) f_{u, j, k}^{ \pm}\left(\gamma^{-1} x\right) \\
& =e^{-2 \pi i u \cdot b} f_{B u}(x) L(\varepsilon(\gamma)) w_{j}^{ \pm} \otimes \rho(\gamma) v_{k} .
\end{aligned}
$$


Let $\gamma_{u, j, k}^{ \pm}:=\left\langle\gamma \cdot f_{u, j, k}^{ \pm}, f_{u, j, k}^{ \pm}\right\rangle=\int_{T_{\Lambda}}\left\langle\gamma \cdot f_{u, j, k}^{ \pm}(x), f_{u, j, k}^{ \pm}(x)\right\rangle d x$. Now, using (2.12) we compute

$$
\begin{aligned}
\gamma_{u, j, k}^{ \pm} & =e^{-2 \pi i u \cdot b} \int_{T_{\Lambda}}\left\langle f_{B u}(x) L(\varepsilon(\gamma)) w_{j}^{ \pm} \otimes \rho(\gamma) v_{k}, f_{u}(x) w_{j}^{ \pm} \otimes v_{k}\right\rangle d x \\
& =e^{-2 \pi i u \cdot b}\left\langle L(\varepsilon(\gamma)) w_{j}^{ \pm} \otimes \rho(\gamma) v_{k}, w_{j}^{ \pm} \otimes v_{k}\right\rangle \int_{T_{\Lambda}} e^{2 \pi i(B u-u) \cdot x} d x \\
& =e^{-2 \pi i u \cdot b}\left\langle L(\varepsilon(\gamma)) w_{j}^{ \pm} \otimes \rho(\gamma) v_{k}, w_{j}^{ \pm} \otimes v_{k}\right\rangle \operatorname{vol}\left(T_{\Lambda}\right) \delta_{B u, u} .
\end{aligned}
$$

In this way we get

$$
\operatorname{tr} p_{\mu}^{ \pm}=\frac{1}{|F|} \sum_{\gamma \in \Lambda \backslash \Gamma} \sum_{u \in\left(\Lambda_{\varepsilon, \mu}^{*}\right)^{B}} \sum_{k=1}^{d_{\rho}}\left\langle\rho(\gamma) v_{k}, v_{k}\right\rangle \sum_{j=1}^{2^{m-1}} e^{-2 \pi i u \cdot b}\left\langle L(\varepsilon(\gamma)) w_{j}^{ \pm}, w_{j}^{ \pm}\right\rangle .
$$

Now, if $\gamma=B L_{b} \in \Gamma$ and $u \in\left(\Lambda_{\varepsilon, \mu}^{*}\right)^{B}$, then $\varepsilon(\gamma) \in \operatorname{Spin}(n-1, u)$ (see (6.6) ). Hence $L(\varepsilon(\gamma))$ preserves the eigenspaces $\mathrm{S}_{u}^{ \pm}$of $L(u) \otimes I d$, and we can consider the trace of $(L \circ \varepsilon \otimes \rho)(\gamma)$ restricted to $\mathrm{S}_{u}^{ \pm} \otimes V$. Thus we finally obtain

$$
d_{\rho, \mu}^{ \pm}(\Gamma, \varepsilon)=\frac{1}{|F|} \sum_{\gamma \in \Lambda \backslash \Gamma} \operatorname{tr} \rho(\gamma) \sum_{u \in\left(\Lambda_{\varepsilon, \mu}^{*}\right)^{B}} e^{-2 \pi i u \cdot b} \operatorname{tr} L(\varepsilon(\gamma))_{\mid S_{u}^{ \pm}} .
$$

The next task will be to compute the traces $\operatorname{tr} L(\varepsilon(\gamma))_{\mid \mathrm{S}_{u}^{ \pm}}$, showing they can be expressed as values of characters of spin representations. The influence of $u$ will only appear in the determination of a sign. We shall use the element $x_{\gamma}$ and the notions introduced in Definition 2.3.

We first note that $\operatorname{tr} L(\varepsilon(\gamma))_{\mid S_{u}^{ \pm}}=\operatorname{tr} L\left(h_{u} \varepsilon(\gamma) h_{u}^{-1}\right)_{\mid S_{e_{n}}^{ \pm}}$. Now, we use Lemma 6.6 together with Definition 2.3. If $n$ is odd and $h_{u} \varepsilon(\gamma) h_{u}^{-1} \sim x_{\gamma}$, then

$$
\operatorname{tr} L\left(h_{u} \varepsilon(\gamma) h_{u}^{-1}\right)_{\mid S_{e_{n}}^{ \pm}}=\operatorname{tr} L_{n-1}^{ \pm}\left(x_{\gamma}\right) .
$$

If $h_{u} \varepsilon(\gamma) h_{u}^{-1} \nsim x_{\gamma}$, then $h_{u} \varepsilon(\gamma) h_{u}^{-1} \sim-e_{1} x_{\gamma} e_{1}$, hence

$$
\operatorname{tr} L\left(h_{u} \varepsilon(\gamma) h_{u}^{-1}\right)_{\mid S_{e_{n}}^{ \pm}}=\operatorname{tr} L_{n-1}^{ \pm}\left(-e_{1} x_{\gamma} e_{1}\right)=\operatorname{tr} L_{n-1}^{\mp}\left(x_{\gamma}\right),
$$

since $L\left(e_{1}\right)$ sends $\mathrm{S}^{ \pm}$to $\mathrm{S}^{\mp}$ orthogonally. For $n$ even we proceed similarly, using (6.3). Thus we obtain

$$
\operatorname{tr} L(\varepsilon(\gamma))_{\mid S_{u}^{ \pm}}= \begin{cases}\operatorname{tr} L_{n-1}\left(x_{\gamma}\right), & n \text { even, } \\ \operatorname{tr} L_{n-1}^{ \pm \sigma\left(u, x_{\gamma}\right)}\left(x_{\gamma}\right), & n \text { odd. }\end{cases}
$$

Substituting (2.14) in (2.13) we get that $d_{\rho, \mu}^{ \pm}(\Gamma, \varepsilon)$ equals

$$
\frac{1}{|F|} \sum_{\gamma \in \Lambda \backslash \Gamma} \chi_{\rho}(\gamma) \sum_{u \in\left(\Lambda_{\varepsilon, \mu}^{*}\right)^{B}} e^{-2 \pi i u \cdot b} \begin{cases}\operatorname{tr} L_{n-1}\left(x_{\gamma}\right), & n \text { even, } \\ \operatorname{tr} L_{n-1}^{ \pm \sigma\left(u, x_{\gamma}\right)}\left(x_{\gamma}\right), & n \text { odd, }\end{cases}
$$

as asserted in formula (2.8) for $n$ even. If $n$ is odd, then, by separating the contributions of the elements $\gamma=B L_{b}$ with $B \in F_{1}$ from those with $B \notin F_{1}$ (see Remark 2.4), we arrive at formula (2.9).

In the case when $\mu=0$ we may proceed in a similar way. If we identify $w \otimes v \in$ $\mathrm{S} \otimes V$ with the constant function $f_{0, w \otimes v}$, then for $\gamma \in \Gamma$ we have

$$
\operatorname{tr} \gamma_{\mid H_{0}}=\frac{1}{\operatorname{vol} T_{\Lambda}} \sum_{j=1}^{2^{m}} \sum_{k=1}^{d_{\rho}}\left\langle\gamma \cdot w_{j} \otimes v_{k}, w_{j} \otimes v_{k}\right\rangle=\chi_{\rho}(\gamma) \chi_{L_{n}}(\varepsilon(\gamma)) .
$$


Thus

$$
\operatorname{dim}\left(H_{0}^{\Gamma}\right)=\frac{1}{|F|} \sum_{\gamma \in \Lambda \backslash \Gamma} \operatorname{tr} \gamma_{\mid H_{0}}=\frac{1}{|F|} \sum_{\gamma \in \Lambda \backslash \Gamma} \chi_{\rho}(\gamma) \chi_{L_{n}}(\varepsilon(\gamma)),
$$

as claimed. Concerning the last equality in the theorem, we know that

$$
H_{0}=\left\{f_{0, w \otimes v}: L \circ \varepsilon \otimes \rho(\gamma) w \otimes v=w \otimes v\right\} \simeq(\mathrm{S} \otimes V)^{F},
$$

since $F \simeq \Lambda \backslash \Gamma$ and $\Lambda$ acts trivially for $\varepsilon$ of trivial type.

Corollary 2.6. Let $\left(M_{\Gamma}, \varepsilon\right)$ be a spin compact flat manifold of dimension $n$. If $n$ is even, or if $n$ is odd and $n_{B}>1$ for every $\gamma=B L_{b} \in \Gamma$ (i.e. $F_{1}=\emptyset$ ), then the spectrum of the twisted Dirac operator $D_{\rho}$ is symmetric.

Proof. If $n$ is even, the assertion is automatic by (2.8). If $n$ is odd, by (2.9) and (6.4) we have that $d_{\rho, \mu}^{+}(\Gamma, \varepsilon)-d_{\rho, \mu}^{-}(\Gamma, \varepsilon)$ equals

$$
\frac{(2 i)^{m}}{|F|} \sum_{\gamma \in \Lambda \backslash \Gamma} \chi_{\rho}(\gamma) e_{\mu, \gamma}\left(\delta_{\varepsilon}\right) \prod_{j=1}^{m} \sin t_{j}\left(x_{\gamma}\right),
$$

where $x_{\gamma}=x\left(t_{1}\left(x_{\gamma}\right), \ldots, t_{m}\left(x_{\gamma}\right)\right)$ in the notation of (1.4). It is clear that $\gamma$ satisfies $n_{B}>1$ if and only if $t_{k}\left(x_{\gamma}\right) \in \pi \mathbb{Z}$ for some $k$, hence in this case $\prod_{j=1}^{m} \sin t_{j}\left(x_{\gamma}\right)=0$. Thus, if $n_{B}>1$ for every $\gamma$, (2.15) implies that the Dirac spectrum is symmetric.

Remark 2.7. In specific examples, the expressions for the multiplicities of eigenvalues in the theorem can be made more explicit by substituting $\chi_{L_{n-1}}\left(x_{\gamma}\right), \chi_{L_{n-1}^{ \pm}}\left(x_{\gamma}\right)$ or $\chi_{L_{n}}(\varepsilon(\gamma))$ by the values given in Lemma 6.1 in terms of products of cosines or sines of the $t_{j}\left(x_{\gamma}\right)$, where $x_{\gamma}=x\left(t_{1}\left(x_{\gamma}\right), \ldots, t_{m}\left(x_{\gamma}\right)\right)$ as above.

Remark 2.8. Set $d_{\rho, \mu}(\Gamma, \varepsilon):=d_{\rho, \mu}^{+}(\Gamma, \varepsilon)+d_{\rho, \mu}^{-}(\Gamma, \varepsilon)$ for $\mu>0$. Note that, since $D_{\rho}^{2}=-\Delta_{s, \rho}$, then $d_{\rho, \mu}(\Gamma, \varepsilon)$ is just the multiplicity of the eigenvalue $4 \pi^{2} \mu^{2}$ of $-\Delta_{s, \rho}$. We thus have

$$
d_{\rho, \mu}(\Gamma, \varepsilon)=\frac{\alpha(n)}{|F|} \sum_{\gamma \in \Lambda \backslash \Gamma} \chi_{\rho}(\gamma) e_{\mu, \gamma}\left(\delta_{\varepsilon}\right) \chi_{L_{n-1}}(\varepsilon(\gamma)),
$$

where $\alpha(n)=1$ or 2 depending on whether $n$ is odd or even. Clearly, (twisted) Dirac isospectrality implies (twisted) spinor Laplacian isospectrality, but we shall see the converse is not true (see Example 4.4).

Spectral asymmetry and $\eta$-series. We decompose $\operatorname{Spec}_{D}\left(M_{\Gamma}, \varepsilon\right)=\mathcal{S} \dot{\mathcal{A}} \mathcal{A}$, where $\mathcal{S}$ and $\mathcal{A}$ are the symmetric and the asymmetric components of the spectrum, respectively. That is, if $\lambda=2 \pi \mu, \lambda \in \mathcal{S}$ if and only if $d_{\rho, \mu}^{+}(\Gamma, \varepsilon)=d_{\rho, \mu}^{-}(\Gamma, \varepsilon)$. We say that $\operatorname{Spec}_{D}\left(M_{\Gamma}, \varepsilon\right)$ is symmetric if $\mathcal{A}=\emptyset$. In this case, the positive spectrum $\operatorname{Spec}_{D}^{+}\left(M_{\Gamma}\right)=\left\{\lambda \in \operatorname{Spec}_{D}\left(M_{\Gamma}\right): \lambda>0\right\}$ and $H_{0}$ determine the whole spectrum $\operatorname{Spec}_{D}\left(M_{\Gamma}\right)$. The symmetry of the spectrum of the Dirac operator depends on $\Gamma$ and also on the spin structure $\varepsilon$ on $M_{\Gamma}$.

Our next goal is to derive an expression for the $\eta$-series $\eta_{(\Gamma, \rho, \varepsilon)}(s)$, for a general Bieberbach manifold $M_{\Gamma}$ with a spin structure $\varepsilon$. Consider

$$
\sum_{\substack{\lambda \in \operatorname{Spec}_{\lambda}\left(M_{\Gamma}, \varepsilon\right) \\ \lambda \neq 0}} \frac{\operatorname{sgn}(\lambda)}{|\lambda|^{s}}=\frac{1}{(2 \pi)^{s}} \sum_{\mu \in \frac{1}{2 \pi} \mathcal{A}} \frac{d_{\rho, \mu}^{+}(\Gamma, \varepsilon)-d_{\rho, \mu}^{-}(\Gamma, \varepsilon)}{|\mu|^{s}} .
$$

It is known that this series converges absolutely for $\operatorname{Re}(s)>n$ and defines a holomorphic function $\eta_{(\Gamma, \rho, \varepsilon)}(s)$ in this region, having a meromorphic continuation to 
$\mathbb{C}$, with simple poles (possibly) at $z=n-k, k \in \mathbb{N}_{0}$, and holomorphic at $s=0$ ([APS] and [Gi]). One defines the eta-invariant of $M_{\Gamma}$ by $\eta_{(\Gamma, \rho, \varepsilon)}:=\eta_{(\Gamma, \rho, \varepsilon)}(0)$. By Corollary 2.6. $\eta(s) \equiv 0$ if $n=2 m$ or if $n=2 m+1$ and $F_{1}=\emptyset$. Furthermore, it is known that if $n \not \equiv 3 \bmod (4)$, then $\eta(s) \equiv 0$ for every Riemannian manifold $M$. In what follows we will often write $\eta(s)$ and $\eta$ in place of $\eta_{(\Gamma, \rho, \varepsilon)}(s)$ and $\eta_{(\Gamma, \rho, \varepsilon)}$, for simplicity.

Proposition 2.9. Let $\Gamma$ be a Bieberbach group of dimension $n=4 r+3$ (thus $m=2 r+1)$ with holonomy group $F$ and let $\varepsilon$ be a spin structure on $M_{\Gamma}=\Gamma \backslash \mathbb{R}^{n}$. Then the $\eta$-series of $M_{\Gamma}$ is given by

$$
\eta_{(\Gamma, \rho, \varepsilon)}(s)=\frac{(2 i)^{m}}{|F|(2 \pi)^{s}} \sum_{\substack{\gamma \in \Lambda \backslash \Gamma \\ B \in F_{1}}} \chi_{\rho}(\gamma)\left(\prod_{j=1}^{m} \sin t_{j}\left(x_{\gamma}\right)\right) \sum_{\mu \in \frac{1}{2 \pi} \mathcal{A}} \frac{e_{\mu, \gamma, \sigma}\left(\delta_{\varepsilon}\right)}{|\mu|^{s}},
$$

where $e_{\mu, \gamma, \sigma}\left(\delta_{\varepsilon}\right)=\sum_{u \in\left(\Lambda_{\varepsilon, \mu}^{*}\right)^{B}} \sigma\left(u, x_{\gamma}\right) e^{-2 \pi i u \cdot b}$ as in (2.7) and, in the notation of (1.4), $x_{\gamma}=x\left(t_{1}\left(x_{\gamma}\right), \ldots, t_{m}\left(x_{\gamma}\right)\right)$.

Proof. By (2.9) and Corollary 2.6, we get that $d_{\rho, \mu}^{+}(\Gamma, \varepsilon)-d_{\rho, \mu}^{-}(\Gamma, \varepsilon)$ equals

$$
\begin{aligned}
& \frac{1}{|F|} \sum_{\substack{\gamma \in \Lambda \backslash \Gamma \\
B \in F_{1}}} \chi_{\rho}(\gamma) \sum_{u \in\left(\Lambda_{\varepsilon, \mu}^{*}\right)^{B}} e^{-2 \pi i u \cdot b}\left(\chi_{L_{n-1}^{\sigma\left(u, x_{\gamma}\right)}}-\chi_{L_{n-1}^{-\sigma\left(u, x_{\gamma}\right)}}\right)\left(x_{\gamma}\right) \\
& =\frac{1}{|F|} \sum_{\substack{\gamma \in \Lambda \backslash \Gamma \\
B \in F_{1}}} \chi_{\rho}(\gamma) \sum_{u \in\left(\Lambda_{\varepsilon, \mu}^{*}\right)^{B}} e^{-2 \pi i u \cdot b} \sigma\left(u, x_{\gamma}\right)\left(\chi_{L_{n-1}^{+}}-\chi_{L_{n-1}^{-}}\right)\left(x_{\gamma}\right) .
\end{aligned}
$$

Now, using (6.4) we get

$$
d_{\rho, \mu}^{+}(\Gamma, \varepsilon)-d_{\rho, \mu}^{-}(\Gamma, \varepsilon)=\frac{(2 i)^{m}}{|F|} \sum_{\substack{\gamma \in \Lambda \backslash \Gamma \\ B \in F_{1}}} \chi_{\rho}(\gamma) e_{\mu, \gamma, \sigma}\left(\delta_{\varepsilon}\right) \prod_{j=1}^{m} \sin t_{j}\left(x_{\gamma}\right) .
$$

By substituting this last expression in (2.17) the proposition follows.

Remark 2.10. (i) In Proposition 3.4 we will give a very explicit expression for the eta series and will compute the eta invariant for a general flat manifold with holonomy group $\mathbb{Z}_{2}^{k}$. We shall see that we may have $\eta=0$ or $\eta \neq 0$, depending on the spin structure.

(ii) Let $g \in \operatorname{Spin}(n)$. If $n$ even, $\chi_{L_{n}^{+}}(g)-\chi_{L_{n}^{-}}(g)=\operatorname{Str} L_{n}(g)$ is the supertrace of $L_{n}(g)$. Furthermore, by Proposition 3.23 in [BGV], one has the expression

$$
\operatorname{Str} L_{n}(g)=i^{-n / 2} \operatorname{sgn}(g)\left|\operatorname{det}\left(I d_{n-1}-\mu(g)\right)\right|^{1 / 2},
$$

where $\operatorname{sgn}(g) \in\{ \pm 1\}$ is defined in [BGV] and $\mu$ is the covering map (1.3).

(iii) Note that some authors use $\frac{1}{2}\left(\eta(0)+d_{0}\right)$ instead of $\eta(0)$ as the definition of the $\eta$-invariant of $(M, \varepsilon)$.

\section{The twisted DiRaC SPeCtrum of $\mathbb{Z}_{2}^{k}$-MAnifolds}

In this section we shall look at the case of $\mathbb{Z}_{2}^{k}$-manifolds, a very rich class of flat manifolds. In this case, the holonomy group $F \simeq \mathbb{Z}_{2}^{k}$, but the holonomy action need not be diagonal in general. In the case when $k=2$ it is already known that there are infinitely many indecomposable holonomy actions, and infinitely many of these give rise to (at least) one Bieberbach group (see [BGR]). Giving a classification for $k \geq 3$ is a problem of wild type. The case $k=n-1$ corresponds to the so-called 
generalized Hantzsche-Wendt manifolds, studied in [MR, RS and MPR. This class is still very rich and will be used in Section 4 to construct large families of Dirac isospectral manifolds pairwise nonhomeomorphic to each other.

If $\Gamma$ has holonomy group $\mathbb{Z}_{2}^{k}$, then $\Gamma=\left\langle\gamma_{1}, \ldots, \gamma_{k}, \Lambda\right\rangle$, where $\gamma_{i}=B_{i} L_{b_{i}}, B_{i} \in$ $\mathrm{O}(n), b_{i} \in \mathbb{R}^{n}, B_{i} \Lambda=\Lambda, B_{i}^{2}=I d$ and $B_{i} B_{j}=B_{j} B_{i}$, for each $1 \leq i, j \leq k$. We shall see that, somewhat surprisingly, for these manifolds the multiplicity formulas in Theorem 2.5 take extremely simple forms (see (3.4) and (3.5)). This will allow us to exhibit, in the next section, large Dirac isospectral sets. Also, we will be able to characterize all manifolds having asymmetric Dirac spectrum and to obtain very explicit expressions for the $\eta$-series and the $\eta$-invariant.

Let $F_{1}$ be as in (1.2). In the case of $\mathbb{Z}_{2}^{k}$-manifolds, $F_{1}$ is the set of $B \in \mathrm{O}(n) \cap r(\Gamma)$ such that $B$ is conjugate in $\mathrm{O}(n)$ to the diagonal matrix $\operatorname{diag}(-1, \ldots,-1,1)$. The next lemma will be very useful in the proof of Theorem 3.2 the main result of this section.

Lemma 3.1. If $\Gamma$ is a Bieberbach group with translation lattice $\Lambda$ and holonomy group $\mathbb{Z}_{2}^{k}$, then the elements in $F_{1}$ can be simultaneously diagonalized in $\Lambda$, that is, there is a basis $f_{1}, \ldots, f_{n}$ of $\Lambda$ such that $B f_{j}= \pm f_{j}, 1 \leq j \leq n$, for any $B L_{b} \in \Gamma$ with $n_{B}=1$. Furthermore $\Gamma$ can be conjugated by some $L_{\mu}, \mu \in \mathbb{R}^{n}$, to a group $\Gamma^{\prime}$ such that $2 b \in \Lambda$ for any $B L_{b} \in \Gamma^{\prime}$ with $n_{B}=1$.

Proof. Let $B L_{b} \in \Gamma$ with $n_{B}=1$. Then $B \Lambda=\Lambda$, and it is a well-known fact that $\Lambda$ decomposes, with respect to the action of $B$, as $\Lambda=\Lambda_{1} \oplus \Lambda_{2}$, where $\Lambda_{1}$ (resp. $\Lambda_{2}$ ) is a direct sum of integral subrepresentations of rank 1 (resp. 2). Here $B$ acts diagonally on $\Lambda_{1}$, whereas $\Lambda_{2}$ is a direct sum of subgroups on which $B$ acts by $J=\left[\begin{array}{ll}0 & 1 \\ 1 & 0\end{array}\right]$. Now, it is not hard to check that since $\Gamma$ is Bieberbach, then the orthogonal projection $2 b^{+} \in \Lambda$ of $2 b$ onto $\left(\mathbb{R}^{n}\right)^{B}$ cannot lie in $\Lambda_{2}$, otherwise some element of the form $B L_{b+\lambda}$, with $\lambda \in \Lambda$, would be of finite order (see [DM], Proposition 2.1). Thus, the multiplicity of the eigenvalue 1 for $B$ is at least 1 on $\Lambda_{1}$. If furthermore $\Lambda_{2} \neq 0$, then it would be at least 1 on $\Lambda_{2}$, hence $n_{B} \geq 2$, which is not possible since $B \in F_{1}$. Thus $\Lambda_{2}=0$ for every $B \in F_{1}$, therefore each such $B$ can be diagonalized in a basis of $\Lambda$ (see Lemma 3.3 in [RS] for a different proof).

Our next task is to show that this can be done simultaneously in $\Lambda$ for all elements in $F_{1}$. To show this, we enumerate the elements in $F_{1}: B_{1}, \ldots, B_{r}$. Let $f_{1}, \ldots, f_{n}$ be a basis of $\Lambda$ diagonalizing $B_{r}$. After reordering we may assume that $B_{r} f_{n}=f_{n}$ and $B_{r} f_{j}=-f_{j}$ for $1 \leq j \leq n-1$. Clearly $f_{n}$ is orthogonal to $f_{j}$ for $j<n$. Now, for $i=1, \ldots, r-1, B_{i} f_{n}= \pm f_{n}$ since $B_{i}$ commutes with $B_{r}$, and actually $B_{i} f_{n}=-f_{n}$; otherwise we would have $B_{i}=B_{r}$, since $B_{i} \in F_{1}$. Now, $B_{1}, \ldots, B_{r-1} \in F_{1}$ and leave $\Lambda^{\prime}:=\mathbb{Z}$-span $\left\{f_{1}, \ldots, f_{n-1}\right\}$ invariant. Hence by induction they can be simultaneously diagonalized in some $\mathbb{Z}$-basis of $\Lambda^{\prime}$. Putting this basis together with $f_{n}$ we get a basis of $\Lambda$ that diagonalizes $B_{1}, \ldots, B_{r}$. The proof of the second assertion now follows in the same way as that of Lemma 1.4 in MR4.

We are now in a position to prove the main result in this section. We shall see that for a spin $\mathbb{Z}_{2}^{k}$-manifold, only the identity element, $I d$, and possibly one element, $B L_{b} \in \Gamma$ with $n_{B}=1$, can give a nonzero contribution to the multiplicity formulas.

We note that given $\gamma=B L_{b} \in \Gamma \backslash \Lambda$, since $B^{2}=I d$, then $B$ is conjugate in $\mathrm{SO}(n)$ to a diagonal matrix of the form $\operatorname{diag}(\underbrace{-1, \ldots,-1}_{2 h}, 1, \ldots, 1)$ with $1 \leq h \leq m$, 
where $m=\left[\frac{n}{2}\right]$, hence $\varepsilon(\gamma)$ is conjugate in $\operatorname{Spin}(n)$ to $\pm g_{h}$ for some $1 \leq h \leq m$, where

$$
g_{h}:=e_{1} \cdots e_{2 h} \in \operatorname{Spin}(n) .
$$

Moreover, if $n=2 m$, then $h<m$, since $B=-I d$ cannot occur for $B L_{b} \in \Gamma, \Gamma$ being torsion-free. Thus, it follows that $g_{h} \in \operatorname{Spin}(n-1)$ and since $g_{h}$ and $-g_{h}$ are conjugate (see Corollary 6.4), then we may take $x_{\gamma}$ in Definition 2.3 to be equal to $g_{h}$, with $h$ depending on $\gamma$. From now on in this section we shall thus assume that $x_{\gamma}=g_{h}$.

We will need the fact that (see Lemma 6.1), if $n=2 m$, then

$$
\chi_{L_{n}^{ \pm}}\left(g_{h}\right)= \begin{cases} \pm 2^{m-1} i^{m}, & h=m, \\ 0, & 1 \leq h<m,\end{cases}
$$

and furthermore, for $n$ even or odd one has

$$
\chi_{L_{n}}\left(g_{h}\right)=0 \quad \text { for any } 1 \leq h \leq m .
$$

Theorem 3.2. Let $\left(M_{\Gamma}, \varepsilon\right)$ be a spin $\mathbb{Z}_{2}^{k}$-manifold of dimension $n$, and let $F_{1}$ be as in (1.2).

(i) If $F_{1}=\emptyset$, then $\operatorname{Spec}_{D_{\rho}}\left(M_{\Gamma}, \varepsilon\right)$ is symmetric and the multiplicities of the eigenvalues $\pm 2 \pi \mu, \mu>0$, of $D_{\rho}$ are given by

$$
d_{\rho, \mu}^{ \pm}(\Gamma, \varepsilon)=2^{m-k-1} d_{\rho}\left|\Lambda_{\varepsilon, \mu}^{*}\right| .
$$

(ii) If $F_{1} \neq \emptyset$, then $\operatorname{Spec}_{D_{\rho}}\left(M_{\Gamma}, \varepsilon\right)$ is asymmetric if and only if the following conditions hold: $n=4 r+3$ and there exists $\gamma=B L_{b}$, with $n_{B}=1$ and $\chi_{\rho}(\gamma) \neq$ 0 , such that $B_{\mid \Lambda}=-\delta_{\varepsilon} I d$. In this case, the asymmetric spectrum is the set of eigenvalues

$$
\mathcal{A}=\operatorname{Spec}_{D_{\rho}}^{A}\left(M_{\Gamma}, \varepsilon\right)=\left\{ \pm 2 \pi \mu_{j}: \mu_{j}=\left(j+\frac{1}{2}\right)\|f\|^{-1}, j \in \mathbb{N}_{0}\right\},
$$

where $\Lambda^{B}=\mathbb{Z} f$, and if we put $\sigma_{\gamma}:=\sigma\left((f \cdot 2 b) f, g_{m}\right)$ we have

$$
d_{\rho, \mu}^{ \pm}(\Gamma, \varepsilon)= \begin{cases}2^{m-k-1}\left(d_{\rho}\left|\Lambda_{\varepsilon, \mu}^{*}\right| \pm 2 \sigma_{\gamma}(-1)^{r+j} \chi_{\rho}(\gamma)\right), & \mu=\mu_{j}, \\ 2^{m-k-1} d_{\rho}\left|\Lambda_{\varepsilon, \mu}^{*}\right|, & \mu \neq \mu_{j} .\end{cases}
$$

If $\operatorname{Spec}_{D_{\rho}}\left(M_{\Gamma}, \varepsilon\right)$ is symmetric, then $d_{\rho, \mu}^{ \pm}(\Gamma, \varepsilon)$ is given by (3.4).

(iii) The number of independent harmonic spinors is given by

$$
d_{\rho, 0}(\Gamma, \varepsilon)= \begin{cases}2^{m-k} d_{\rho}, & \text { if } \varepsilon_{\mid \Lambda}=1 \\ 0, & \text { otherwise }\end{cases}
$$

If $k>m$, then $M_{\Gamma}$ has no spin structures of trivial type, hence $M_{\Gamma}$ has no harmonic spinors. Furthermore, if $M_{\Gamma}$ has exactly $2^{m} d_{\rho}$ harmonic spinors, then $M_{\Gamma}=T_{\Lambda}$ and $\varepsilon=1$.

Proof. We first note that the contribution of $I d \in F$ to the multiplicity formulas (2.8) and (2.9) is given by $2^{m-k-1} d_{\rho} e_{\mu, I d}\left(\delta_{\varepsilon}\right)=2^{m-k-1} d_{\rho}\left|\Lambda_{\varepsilon, \mu}^{*}\right|$. Hence, when no element in $F$ other than Id gives a nonzero contribution, then (3.4) holds.

If $F_{1}=\emptyset$, then, for any $\gamma=B L_{b} \in \Gamma \backslash \Lambda$, we have $x_{\gamma}=g_{h}$ with $h<m$. Thus, $\chi_{L_{n}}\left(x_{\gamma}\right)=0$ if $n$ is odd and $\chi_{L_{n}}^{ \pm}\left(x_{\gamma}\right)=0$ if $n$ is even, for any $\gamma \in \Gamma \backslash \Lambda$. Thus, in this case, only $I d \in F$ contributes to (2.8) and (2.9), and hence (i) follows. This implies that, other than $I d$, only the elements in $F_{1}$ can give a nonzero contribution to the multiplicities and furthermore, this can happen only if $n$ is odd. 
Now, assume that $n=2 m+1$ and $F_{1} \neq \emptyset$. If $\gamma=B L_{b} \in \Gamma \backslash \Lambda$, with $n_{B}=1$ (hence $x_{\gamma}=g_{m}$ ), we know by Lemma 3.1 that there exists a basis $f_{1}, \ldots, f_{n}$ of $\Lambda$ such that $B$ is diagonal in this basis. After reordering the basis elements we may assume that

$$
B f_{j}=-f_{j} \text { for } 1 \leq j<n, \quad B f_{n}=f_{n}, \quad b \equiv \frac{1}{2} f_{n} \quad \bmod \Lambda .
$$

Let $f_{1}^{\prime}, \ldots, f_{n}^{\prime} \in \Lambda^{*}$ be the dual basis of $f_{1}, \ldots, f_{n}$. It is also clear that $B f_{j}^{\prime}=-f_{j}^{\prime}$ for $1 \leq j<n$ and $f_{n}^{\prime}=\frac{f_{n}}{\left\|f_{n}\right\|^{2}}$, thus $B f_{n}^{\prime}=f_{n}^{\prime}$.

Let as usual $\Lambda_{\varepsilon}^{*}=\Lambda^{*}+u_{\varepsilon}$, with $u_{\varepsilon}=\sum_{j} c_{j} f_{j}^{\prime}$ and $c_{j} \in\left\{0, \frac{1}{2}\right\}$ for each $j$. If the contribution of $B$ to (2.9) is nontrivial, then $\left(\Lambda_{\varepsilon}^{*}\right)^{B} \neq \emptyset$. Thus, there exists $u=\lambda^{\prime}+u_{\varepsilon}$ with $\lambda^{\prime}=\sum_{j} d_{j} f_{j}^{\prime}, d_{j} \in \mathbb{Z}$, and such that $B u=u$. This says that for $1 \leq j \leq n-1$, we have $c_{j}+d_{j}=-c_{j}-d_{j}$, and hence $c_{j}=0$ for $1 \leq j \leq n-1$. On the other hand, $c_{n}$ equals 0 or $\frac{1}{2}$, that is, $u_{\varepsilon}=0$ or $u_{\varepsilon}=\frac{1}{2} f_{n}^{\prime}$.

If $u_{\varepsilon}=0$ we have $\Lambda_{\varepsilon}^{*}=\Lambda^{*}$ and thus $e^{2 \pi i u \cdot b}=e^{-2 \pi i u \cdot b}$ for any $u \in \Lambda^{*}$, since $b \in \frac{1}{2} \Lambda$, by Lemma 3.1. We claim that $\sum_{u} e^{-2 \pi i u \cdot b} \chi_{L_{n-1}^{ \pm \sigma\left(u, x_{\gamma}\right)}}\left(x_{\gamma}\right)=0$, where the sum is taken over $u \in\left(\Lambda_{\varepsilon, \mu}^{*}\right)^{B}$. Indeed, putting together the contributions of $u$ and $-u$ in the expression above, by Remark 2.4 and (3.3) we get

$$
e^{-2 \pi i u \cdot b}\left(\chi_{L_{n-1}^{ \pm \sigma\left(u, g_{m}\right)}}+\chi_{L_{n-1}^{ \pm \sigma\left(-u, g_{m}\right)}}\right)\left(g_{m}\right)=e^{-2 \pi i u \cdot b} \sigma\left(u, g_{m}\right) \chi_{L_{n-1}}\left(g_{m}\right)=0 .
$$

Hence, we conclude that if $u_{\varepsilon}=0$, the contribution of $\gamma=B L_{b} \in \Gamma \backslash \Lambda$ to (2.9) is zero.

Now consider the case $u_{\varepsilon}=\frac{1}{2} f_{n}^{\prime}$, that is, $\left(\Lambda_{\varepsilon}^{*}\right)^{B}=\left(\mathbb{Z}+\frac{1}{2}\right) f_{n}^{\prime}$. Hence, since $\delta_{\varepsilon}(\lambda)=e^{2 \pi i u_{\varepsilon} \cdot \lambda}$, then

$$
\varepsilon\left(L_{f_{j}}\right)=1 \quad(1 \leq j \leq n-1), \quad \text { and } \quad \varepsilon\left(L_{f_{n}}\right)=-1 .
$$

Furthermore we note that, since

$$
-1=\varepsilon\left(L_{f_{n}}\right)=\varepsilon\left(\gamma^{2}\right)=\varepsilon(\gamma)^{2}=\left( \pm e_{1} \ldots e_{2 m}\right)^{2}=(-1)^{m},
$$

it follows that $m$ is necessarily odd, hence $m=2 r+1$ and $n=4 r+3$.

Now (3.6) and (3.7) say that only if $B_{\mid \Lambda}=-\delta_{\varepsilon} I d$ can $\gamma=B L_{b}$ give a nonzero contribution.

Furthermore, since $\left(\Lambda_{\varepsilon}^{*}\right)^{B}=\left(\mathbb{Z}+\frac{1}{2}\right) f_{n}^{\prime}$, then, for fixed $\mu>0,\left(\Lambda_{\varepsilon, \mu}^{*}\right)^{B} \neq \emptyset$ if and only if $\mu=\mu_{j}:=\left(j+\frac{1}{2}\right)\left\|f_{n}\right\|^{-1}$ with $j \in \mathbb{N}_{0}$. For $\mu=\mu_{j}$, we have that $\left(\Lambda_{\varepsilon, \mu_{j}}^{*}\right)^{B}=\left\{ \pm u_{j}\right\}$, where $u_{j}=\left(j+\frac{1}{2}\right) f_{n}^{\prime}$ with $j=\left\|f_{n}\right\| \mu_{j}-\frac{1}{2} \in \mathbb{N}_{0}$. Again, putting together the contributions of $u_{j}$ and $-u_{j}$, we get that the sum over $\left(\Lambda_{\varepsilon, \mu}^{*}\right)^{B}$ in (2.9) equals

$$
\begin{aligned}
& e^{-2 \pi i u_{j} \cdot b} \chi_{L_{n-1}^{ \pm \sigma\left(u_{j}, g_{m}\right)}}\left(g_{m}\right)+e^{2 \pi i u_{j} \cdot b} \chi_{L_{n-1}^{ \pm \sigma\left(-u_{j}, g_{m}\right)}}\left(g_{m}\right) \\
= & \left(e^{-2 \pi i u_{j} \cdot b}-e^{2 \pi i u_{j} \cdot b}\right) \sigma\left(u_{j}, g_{m}\right) \chi_{L_{n-1}^{ \pm}}\left(g_{m}\right) \\
= & \mp 2^{m} i^{m+1} \sigma\left(u_{j}, g_{m}\right) \sin \left(2 \pi u_{j} \cdot b\right),
\end{aligned}
$$

where we have used that $\chi_{L_{n-1}^{-}}\left(g_{h}\right)=-\chi_{L_{n-1}^{+}}\left(g_{h}\right)$ by (3.2). If $\Lambda^{B}=\mathbb{Z} f$, then one has that $f= \pm f_{n}$. Now one verifies that $\sigma\left(u_{j}, g_{m}\right)=\sigma\left((f \cdot 2 b) f, g_{m}\right)=\sigma_{\gamma}$. Hence,

$$
\sigma\left(u_{j}, g_{m}\right) \sin \left(2 \pi u_{j} \cdot b\right)=\sigma_{\gamma} \sin \left(\pi\left(j+\frac{1}{2}\right)\right)=\sigma_{\gamma}(-1)^{j}
$$

since $b \equiv \frac{1}{2} f_{n} \bmod \Lambda$. 
Since $m=2 r+1$, we finally get that the contribution of $\gamma$ to the multiplicity of the eigenvalue $\pm 2 \pi \mu_{j}$ is given by

$$
\pm 2^{m-k} \sigma_{\gamma}(-1)^{r+j} \chi_{\rho}(\gamma)
$$

The above shows that if an element $\gamma^{\prime}=B^{\prime} L_{b^{\prime}} \in F_{1}$ gives a nonzero contribution, then $B_{\mid \Lambda}^{\prime}=-\delta_{\varepsilon} I d$, hence $B^{\prime}=B$, and $b^{\prime} \equiv \frac{1}{2} f_{n}$, $\bmod \Lambda$. Since only $I d$ and $\gamma=B L_{b}$ give a contribution to the multiplicity formula, this completes the proof of (ii).

Finally, the first assertion in (iii) follows immediately from (2.10), and the remaining assertions are direct consequences of the first.

Remark 3.3. (i) Except for the very special case described in (ii) of the theorem, the twisted Dirac spectrum of $\mathbb{Z}_{2}^{k}$-manifolds is symmetric, and the multiplicities are given by the simple formula (3.4). In this case, the multiplicities of Dirac eigenvalues for $M_{\Gamma}$ with a spin structure $\varepsilon$ are determined by the multiplicities for the covering torus $T_{\Lambda}$ with the restricted spin structure $\varepsilon_{\mid \Lambda}$. Indeed, we have

$$
d_{\rho, \mu}^{ \pm}(\Gamma, \varepsilon)=2^{-k} d_{\rho, \mu}^{ \pm}\left(\Lambda, \varepsilon_{\mid \Lambda}\right) .
$$

(ii) Note that, for each fixed $\rho$, all spin $\mathbb{Z}_{2}^{k}$-manifolds $\left(M_{\Gamma}, \varepsilon\right)$ having asymmetric Dirac spectrum and having the same covering torus $\left(T_{\Lambda}, \varepsilon_{\mid \Lambda}\right)$ are $D_{\rho}$-isospectral to each other.

As an application of Theorem 3.2 we shall now compute the $\eta$-series and the $\eta$-invariant for any $\mathbb{Z}_{2}^{k}$-manifold.

Proposition 3.4. Let $\left(M_{\Gamma}, \varepsilon\right)$ be a spin $\mathbb{Z}_{2}^{k}$-manifold of odd dimension $n=4 r+3$ (thus $m=2 r+1$ ). If $\operatorname{Spec}_{D_{\rho}}\left(M_{\Gamma}, \varepsilon\right)$ is asymmetric, then, in the notation of Theorem 3.2, we have

$$
\eta_{(\Gamma, \rho, \varepsilon)}(s)=(-1)^{r} \sigma_{\gamma} \chi_{\rho}(\gamma) 2^{m-k+1} \frac{\|f\|^{s}}{(4 \pi)^{s}}\left(\zeta\left(s, \frac{1}{4}\right)-\zeta\left(s, \frac{3}{4}\right)\right),
$$

where $\zeta(s, \alpha)=\sum_{j=0}^{\infty} \frac{1}{(j+\alpha)^{s}}$ denotes the Hurwitz zeta function for $\alpha \in(0,1]$ and $\sigma_{\gamma} \in\{ \pm 1\}$ is as defined in (ii) of Theorem 3.2 .

Therefore, $\eta_{(\Gamma, \rho, \varepsilon)}(s)$ has an analytic continuation to $\mathbb{C}$ that is everywhere holomorphic. Furthermore,

$$
\begin{aligned}
& \eta_{(\Gamma, \rho, \varepsilon)}(0)=(-1)^{r} \sigma_{\gamma} \chi_{\rho}(\gamma) 2^{m-k} \\
& \eta_{(\Gamma, \rho, \varepsilon)}^{\prime}(0)=\left(4 \log \Gamma\left(\frac{1}{4}\right)+\log \|f\|-3 \log (2 \pi)\right) \eta(0) .
\end{aligned}
$$

Proof. We shall use Theorem 3.2 in the case of the special spin structure when the spectrum is not symmetric, otherwise, $\eta(s)=0$. We have

$$
\eta_{(\Gamma, \rho, \varepsilon)}(s)=\frac{1}{(2 \pi)^{s}} \sum_{j=0}^{\infty} \frac{d_{\rho, \mu_{j}}^{+}(\Gamma, \varepsilon)-d_{\rho, \mu_{j}}^{-}(\Gamma, \varepsilon)}{\left|\mu_{j}\right|^{s}} .
$$

Now from formula (3.5) we have that

$$
d_{\rho, \mu_{j}}^{+}(\Gamma, \varepsilon)-d_{\rho, \mu_{j}}^{-}(\Gamma, \varepsilon)=(-1)^{r+j} \sigma_{\gamma} \chi_{\rho}(\gamma) 2^{m-k+1} .
$$


Thus, if $\operatorname{Re}(s)>n$

$$
\begin{aligned}
\eta_{(\Gamma, \rho, \varepsilon)}(s) & =(-1)^{r} \sigma_{\gamma} \chi_{\rho}(\gamma) 2^{m-k+1} \frac{\|f\|^{s}}{(2 \pi)^{s}} \sum_{j=0}^{\infty} \frac{(-1)^{j}}{\left(j+\frac{1}{2}\right)^{s}} \\
& =(-1)^{r} \sigma_{\gamma} \chi_{\rho}(\gamma) 2^{m-k+1} \frac{\left\|\frac{1}{2} f\right\|^{s}}{(2 \pi)^{s}}\left(\sum_{j=0}^{\infty} \frac{1}{\left(j+\frac{1}{4}\right)^{s}}-\sum_{j=0}^{\infty} \frac{1}{\left(j+\frac{3}{4}\right)^{s}}\right) \\
& =(-1)^{r} \sigma_{\gamma} \chi_{\rho}(\gamma) 2^{m-k+1} \frac{\left\|\frac{1}{2} f\right\|^{s}}{(2 \pi)^{s}}\left(\zeta\left(s, \frac{1}{4}\right)-\zeta\left(s, \frac{3}{4}\right)\right),
\end{aligned}
$$

where $\zeta(s, \alpha)$ is the Hurwitz zeta function, for $\alpha \in(0,1]$. Now $\zeta(s, \alpha)$ extends to an everywhere holomorphic function except for a simple pole at $s=1$, with residue 1 (see [WW, 13.13) hence formula (3.8) implies that $\eta(s)$ is everywhere holomorphic.

Furthermore, since $\zeta(0, \alpha)=\frac{1}{2}-\alpha$, by taking the limit as $s \rightarrow 0$ in the above expression we get (3.9). Also, differentiating (3.8) and using that $\zeta^{\prime}(0, a)=$ $\log \Gamma(a)-\frac{1}{2} \log (2 \pi)$ (see [WW] we obtain (3.10).

Remark 3.5. Note that if $(\rho, V)=(1, \mathbb{C})$, then for $\mathbb{Z}_{2}^{k}$-manifolds with $k \leq m$, one has that $\eta(0) \in 2 \mathbb{Z}$. In particular the $\eta$-invariant of any $\mathbb{Z}_{2}$-manifold is an even integer. Indeed, in the asymmetric case, $\eta= \pm 2^{m-1} \in \mathbb{Z}$. In dimension $n=3$, for $F \simeq \mathbb{Z}_{2}$, the proposition gives $\eta=\sigma_{\gamma}$. Take $\Gamma=\left\langle\gamma, L_{\Lambda}\right\rangle$ where $\gamma=\left[\begin{array}{lll}-1 & & \\ & -1 & \\ & & \end{array}\right] L_{\frac{e_{3}}{2}}$ and $\Lambda$ is the canonical lattice in $\mathbb{R}^{3}$. Then $M_{\Gamma}$ has asymmetric spectrum only for the two spin structures $\varepsilon_{+}=\left(1,1,-1 ; e_{1} e_{2}\right)$ and $\varepsilon_{-}=\left(1,1,-1 ;-e_{1} e_{2}\right)$. We have $\eta_{\left(\Gamma, \varepsilon_{+}\right)}=1$ while $\eta_{\left(\Gamma, \varepsilon_{-}\right)}=-1$ as in [Pf].

\section{Dirac isospectral manifolds}

In this section we give examples of twisted Dirac isospectral flat manifolds that are pairwise nonhomeomorphic to each other. In Examples 4.3, 4.4 and 4.5 we compare (twisted) Dirac isospectrality with other types of isospectrality, such as Laplace isospectrality on functions and on $p$-forms and length isospectrality with and without multiplicities (see the Introduction). Two manifolds are $[L]$-isospectral ( $L$-isospectral) if they have the same $[L]$-spectrum ( $L$-spectrum). Obviously, $[L]$ isospectrality implies $L$-isospectrality.

As a consequence we will obtain the following results.

Theorem 4.1. (i) There are families $\mathcal{F}$ of pairwise nonhomeomorphic Riemannian manifolds mutually twisted Dirac isospectral that are neither Laplace isospectral on functions nor $L$-isospectral. Furthermore, $\mathcal{F}$ can be chosen so that:

(a) Every $M \in \mathcal{F}$ has (no) harmonic spinors (Example 4.3(i)).

(b) All $M$ 's in $\mathcal{F}$ have the same $p$-Betti numbers for $1 \leq p \leq n$, and they are p-isospectral to each other for any $p$ odd (Example 4.3(ii)).

(ii) There are pairs of nonhomeomorphic spin manifolds that are $\Delta_{s, \rho}$-isospectral but not $D_{\rho}$-isospectral (Example 4.4(ii)).

(iii) There are pairs of spin manifolds that are $\Delta_{p}$-isospectral for $0 \leq p \leq n$ and also $[L]$-isospectral, and that are $D_{\rho}$-isospectral, or not, depending on the spin structure (Example 4.5(i)).

(iv) There are pairs of spin manifolds that are $D_{\rho}$-isospectral and $\Delta_{p}$-isospectral for $0 \leq p \leq n$ which are $L$-isospectral but not $[L]$-isospectral (Example 4.5(ii)). 
Theorem 4.2. There exists a family, with cardinality depending exponentially on $n\left(\right.$ or $\left.n^{2}\right)$, of pairwise nonhomeomorphic Kähler Riemannian n-manifolds, that are twisted Dirac isospectral to each other for many different spin structures (Example 4.6, Remark 4.7).

To construct the examples, it will suffice to work with flat $n$-manifolds of diagonal type, having holonomy group $\mathbb{Z}_{2}^{k}, k=1,2$ and $n-1$.

A Bieberbach group $\Gamma$ is said to be of diagonal type if there exists an orthonormal $\mathbb{Z}$-basis $\left\{e_{1}, \ldots, e_{n}\right\}$ of the lattice $\Lambda$ such that for any element $B L_{b} \in \Gamma, B e_{i}= \pm e_{i}$ for $1 \leq i \leq n$ (see [MR4]). Similarly, $M_{\Gamma}$ is said to be of diagonal type, if $\Gamma$ is so. If $\Gamma$ is of diagonal type, after conjugation of $\Gamma$ by an isometry, it may be assumed that $\Lambda$ is the canonical lattice and also that for any $\gamma=B L_{b} \in \Gamma$, one has that $b \in \frac{1}{2} \Lambda$ (MR4, Lemma 1.4).

Let $\left(M_{\Gamma}, \varepsilon\right)$ be a spin $\mathbb{Z}_{2}^{k}$-manifold where $\Gamma=\left\langle\gamma_{1}, \ldots, \gamma_{k}, L_{\Lambda}\right\rangle$ and let $\lambda_{1}, \ldots \lambda_{n}$ be a $\mathbb{Z}$-basis of $\Lambda$. If $\gamma=B L_{b} \in \Gamma$ we will fix a distinguished (though arbitrary) element in $\mu^{-1}(B)$, denoted by $u(B)$. Thus, $\varepsilon(\gamma)=\sigma u(B)$, where $\sigma \in\{ \pm 1\}$ depends on $\gamma$ and on the choice of $u(B)$.

The homomorphism $\varepsilon: \Gamma \rightarrow \operatorname{Spin}(n)$ is determined by its action on the generators $\lambda_{1}, \ldots, \lambda_{n}, \gamma_{1}, \ldots, \gamma_{k}$ of $\Gamma$. Hence, if we set $\delta_{i}:=\varepsilon\left(L_{\lambda_{i}}\right)$, we may identify the spin structure $\varepsilon$ with the $n+k$-tuple

$$
\left(\delta_{1}, \ldots, \delta_{n}, \sigma_{1} u\left(B_{1}\right), \ldots, \sigma_{k} u\left(B_{k}\right)\right),
$$

where $\sigma_{i}$ is defined by the equation $\varepsilon\left(\gamma_{i}\right)=\sigma_{i} u\left(B_{i}\right)$, for $1 \leq i \leq k$.

Example 4.3 ( $\mathbb{Z}_{2}$-manifolds). Here we will give some large twisted Dirac isospectral sets of $\mathbb{Z}_{2}$-manifolds. Put $J:=\left[\begin{array}{ll}0 & 1 \\ 1 & 0\end{array}\right]$. For each $0 \leq j, h<n$, define

$$
B_{j, h}:=\operatorname{diag}(\underbrace{J, \ldots, J}_{j}, \underbrace{-1, \ldots,-1}_{h}, \underbrace{1, \ldots, 1}_{l}),
$$

where $n=2 j+h+l, j+h \neq 0$ and $l \geq 1$. Then $B_{j, h} \in \mathrm{O}(n), B_{j, h}^{2}=I d$ and $B_{j, h} \in \mathrm{SO}(n)$ if and only if $j+h$ is even. Let $\Lambda=\mathbb{Z} e_{1} \oplus \ldots \oplus \mathbb{Z} e_{n}$ be the canonical lattice of $\mathbb{R}^{n}$, and for $j, h$ as before define the groups

$$
\Gamma_{j, h}:=\left\langle B_{j, h} L_{\frac{e_{n}}{2}}, \Lambda\right\rangle .
$$

We have that $\left(B_{j, h}+I d\right) \frac{e_{n}}{2}=e_{n} \in \Lambda \backslash\left(B_{j, h}+I d\right) \Lambda$. Hence, by Proposition 2.1 in DM], the $\Gamma_{j, h}$ are Bieberbach groups. In this way, if we set $M_{j, h}=\Gamma_{j, h} \backslash \mathbb{R}^{n}$, we have a family

$$
\mathcal{F}=\left\{M_{j, h}=\Gamma_{j, h} \backslash \mathbb{R}^{n}: 0 \leq j \leq\left[\frac{n-1}{2}\right], 0 \leq h<n-2 j, j+h \neq 0\right\}
$$

of compact flat manifolds with holonomy group $F \simeq \mathbb{Z}_{2}$. The family $\mathcal{F}$ gives a system of representatives for the diffeomorphism classes of $\mathbb{Z}_{2}$-manifolds of dimension $n$ (see Proposition 4.2 in $[\mathrm{MP}]$ ).

We will make use of the following result on the existence of spin structures (see Proposition 4.2 in $\mathrm{MP}$, and also Theorem 2.1 and Lemma 3.1):

$M_{j, h}$ has $2^{n-j}$ spin structures parametrized by the tuples $\left(\delta_{1}, \ldots, \delta_{n}, \sigma\right) \in\{ \pm 1\}^{n+1}$ satisfying:

$$
\delta_{1}=\delta_{2}, \cdots, \delta_{2 j-1}=\delta_{2 j} \quad \text { and } \quad \delta_{n}=(-1)^{\frac{j+h}{2}} .
$$


Isospectrality on p-forms. We first review from MR3 and MR4 some results on spectra of Laplace operators on vector bundles over flat manifolds. If $\tau$ is a finitedimensional representation of $K=\mathrm{O}(n)$ and $G=I\left(\mathbb{R}^{n}\right)$, we form the vector bundle $E_{\tau}$ over $G / K \simeq \mathbb{R}^{n}$ associated to $\tau$ and consider the corresponding vector bundle $\Gamma \backslash E_{\tau}$ over $\Gamma \backslash \mathbb{R}^{n}=M_{\Gamma}$. As usual we denote by $\chi_{\tau}$ and $d_{\tau}$ respectively, the character and the dimension of $\tau$. Let $-\Delta_{\tau}$ be the connection Laplacian on this bundle.

We recall from [MR4], Theorem 2.1, that the multiplicity of the eigenvalue $4 \pi^{2} \mu$ of $-\Delta_{\tau}$ is given by

$$
d_{\tau, \mu}(\Gamma)=\frac{1}{|F|} \sum_{\gamma=B L_{b} \in \Lambda \backslash \Gamma} \chi_{\tau}(B) e_{\mu, \gamma} \text { where } e_{\mu, \gamma}=\sum_{\substack{v \in\left(\Lambda^{*}\right)^{B} \\\|v\|^{2}=\mu}} e^{-2 \pi i v \cdot b} .
$$

If $\tau=\tau_{p}$, the $p$-exterior representation of $\mathrm{O}(n)$, then $-\Delta_{\tau_{p}}$ corresponds to the Hodge Laplacian acting on $p$-forms. In this case we shall write $\Delta_{p}, \operatorname{tr}_{p}(B)$ and $d_{p, \mu}(\Gamma)$ in place of $\Delta_{\tau_{p}}, \operatorname{tr} \tau_{p}(B)$ and $d_{\tau_{p}, \mu}(\Gamma)$, respectively.

Thus, the $p$-Laplacian $\Delta_{p}, 0 \leq p \leq n$, has eigenvalues $4 \pi^{2} \mu$ with multiplicities $d_{p, \mu}$ given by formula (4.6). Furthermore, for flat manifolds of diagonal type the traces $\operatorname{tr}_{p}(B)$ are given by integral values of the Krawtchouk polynomials $K_{p}^{n}(x)$ (see [MR3, Remark 3.6, and also MR4]). Indeed, we have

$$
\operatorname{tr}_{p}(B)=K_{p}^{n}\left(n-n_{B}\right), \quad \text { where } K_{p}^{n}(x):=\sum_{t=0}^{p}(-1)^{t}\left(\begin{array}{c}
x \\
t
\end{array}\right)\left(\begin{array}{c}
n-x \\
p-t
\end{array}\right) .
$$

Note that $B_{j, h}$ and $B_{0, j+h}$ are conjugate in $\operatorname{GL}(n, \mathbb{R})$, hence

$$
\operatorname{tr}_{p}\left(B_{j, h}\right)=\operatorname{tr}_{p}\left(B_{0, j+h}\right)=K_{p}^{n}(j+h) .
$$

Thus,

$$
d_{p, \mu}\left(\Gamma_{j, h}\right)=\frac{1}{2}\left(\left(\begin{array}{l}
n \\
p
\end{array}\right)\left|\Lambda_{\sqrt{\mu}}\right|+K_{p}^{n}(j+h) e_{\mu, \gamma}\left(\Gamma_{j, h}\right)\right) .
$$

Hence, the existence of integral zeros of $K_{p}^{n}(x)$ will imply $p$-isospectrality of some of the $M_{j, h} \in \mathcal{F}$. For some facts on integral zeros of $K_{p}^{n}(x)$ see [KL, p. 76, or Lemma 3.9 in [MR4]. The simplest are the so-called trivial zeros, namely:

If $n$ is even, then $K_{\frac{n}{2}}^{n}(j)=K_{j}^{n}\left(\frac{n}{2}\right)=0$ for any $j$ odd. Also, $K_{k}^{n}(j)=0$ if and only if $K_{j}^{n}(k)=0$.

Thus for $n=2 m$, all manifolds $\left\{M_{j, h}: j+h\right.$ is odd $\}$ are $m$-isospectral and all manifolds $\left\{M_{j, h}: j+h=m\right\}$ are $p$-isospectral for any $p$ odd with $1 \leq p \leq n$. However, generically, i.e., for arbitrary $p$ and $n$, the $M_{j, h}$ will not be $p$-isospectral to each other because the integral roots of Krawtchouk polynomials, aside from the trivial zeros, are very sporadic.

We claim that the manifolds in $\mathcal{F}$ are pairwise not isospectral on functions. Take $\mu=1$. Then $\Lambda_{1}=\left\{ \pm e_{1}, \ldots, \pm e_{n}\right\}$ and $\Lambda_{1}^{B_{j, h}}=\left\{ \pm e_{2 j+h+1}, \ldots, \pm e_{n}\right\}$, thus $\left|\Lambda_{1}\right|=2 n$ and $\left|\Lambda_{1}^{B, h}\right|=2(n-(2 j+h))=2 l$. Now, one checks that $e_{1, \gamma}\left(\Gamma_{j, h}\right)=$ $2(l-1)+2(-1)=2(l-2)$ and hence, from (4.8), we get

$$
d_{p, 1}\left(\Gamma_{j, h}\right)=\left(\begin{array}{l}
n \\
p
\end{array}\right) n+K_{p}^{n}(j+h)(l-2) .
$$

Now consider $\mu=2$. Then $\Lambda_{\sqrt{2}}=\left\{ \pm\left(e_{i} \pm e_{j}\right): 1 \leq i<j \leq n\right\}$ and $\Lambda_{\sqrt{2}}^{B_{j, h}}=$ $\left\{ \pm\left(e_{2 i-1}+e_{2 i}\right): 1 \leq i \leq j\right\} \cup\left\{ \pm\left(e_{i} \pm e_{j}\right): l+1 \leq i<j \leq n\right\}$. Hence $\left|\Lambda_{\sqrt{2}}\right|=4\left(\begin{array}{c}n \\ 2\end{array}\right)$ 
and $\left|\Lambda_{\sqrt{2}}^{B_{j, h}}\right|=2 j+4\left(\begin{array}{l}l \\ 2\end{array}\right)$. One checks that $e_{2, \gamma}\left(\Gamma_{j, h}\right)=2 j-4(l-1)+\left(4\left(\begin{array}{l}l \\ 2\end{array}\right)-4(l-1)\right)=$ $2 j+2(l-1)(l-4)$. Again by (4.8), we get

$$
d_{p, 2}\left(\Gamma_{j, h}\right)=2\left(\begin{array}{l}
n \\
p
\end{array}\right)\left(\begin{array}{l}
n \\
2
\end{array}\right)+K_{p}^{n}(j+h)(j+(l-1)(l-4)) .
$$

In particular for $p=0$, since $K_{0}^{n}(j)=1$ for any $j$, we have

$$
\begin{aligned}
& d_{0,1}\left(\Gamma_{j, h}\right)=n+l-2, \\
& d_{0,2}\left(\Gamma_{j, h}\right)=n(n-1)+j+(l-1)(l-4) .
\end{aligned}
$$

These multiplicities are sufficient to show that all $\mathbb{Z}_{2}$-manifolds in $\mathcal{F}$ are pairwise not isospectral. Indeed, if $M_{j, h}, M_{j^{\prime}, h^{\prime}}$ are isospectral, then $l=l^{\prime}$ by (4.11), thus $2 j+h=2 j^{\prime}+h^{\prime}$. By (4.12), then $j=j^{\prime}$ and hence $h=h^{\prime}$.

Dirac isospectrality. We will give families of spin $\mathbb{Z}_{2}$-manifolds Dirac isospectral to each other.

We need to restrict ourselves to orientable manifolds, so consider the family

$$
\mathcal{F}^{+}=\left\{M_{j, h} \in \mathcal{F}: j+h \text { is even }\right\} .
$$

It will also be convenient to split $\mathcal{F}^{+}=\mathcal{F}_{0}^{+} \dot{\cup} \mathcal{F}_{1}^{+}$, where

$$
\mathcal{F}_{i}^{+}=\left\{M_{j, h} \in \mathcal{F}^{+}: j+h \equiv 2 i \bmod (4)\right\}, \quad i=0,1 .
$$

(i) We now define spin structures for $M_{j, h}$ in $\mathcal{F}^{+}$. By (4.5), $\delta_{n}=1$ for $j+h \equiv 0$ (4) and $\delta_{n}=-1$ for $j+h \equiv 2(4)$. Hence, we take the spin structures

$$
\varepsilon_{i, j, h}=\left(1, \ldots, 1,(-1)^{i} ; \sigma u\left(B_{j, h}\right)\right), \quad i=0,1,
$$

for manifolds in $\mathcal{F}_{i}^{+}, i=0,1$, respectively. For simplicity, we will write $\varepsilon_{i}$ for $\varepsilon_{i, j, h}$.

We claim that all the spin $\mathbb{Z}_{2}$-manifolds in $\tilde{\mathcal{F}}_{0}^{+}:=\left\{\left(M_{j, h}, \varepsilon_{0}\right): M_{j, h} \in \mathcal{F}_{0}^{+}\right\}$ are twisted Dirac isospectral to each other. Indeed, since $\varepsilon_{0}$ is a spin structure of trivial type, we know from Theorem 3.2 that the spectrum is symmetric and the multiplicities of the eigenvalues $\pm 2 \pi \mu$ of $D_{\rho}$ are given by

$$
d_{\rho, \mu}^{ \pm}\left(\Gamma_{j, h}, \varepsilon_{0}\right)=2^{m-2} d_{\rho}\left|\Lambda_{\varepsilon_{0}, \mu}^{*}\right|=2^{m-2} d_{\rho}\left|\Lambda_{\mu}\right|
$$

since $\Lambda_{\varepsilon_{0}}^{*}=\Lambda$. Note that all manifolds in $\tilde{\mathcal{F}}_{0}^{+}$have $2^{m-1} d_{\rho}$ nontrivial harmonic spinors.

If $n \not \equiv 3(4)$, the spin manifolds in $\tilde{\mathcal{F}}_{1}^{+}:=\left\{\left(M_{j, h}, \varepsilon_{1}\right): M_{j, h} \in \mathcal{F}_{1}^{+}\right\}$are Dirac isospectral to each other. The same happens with those in $\tilde{\mathcal{F}}_{1}^{+} \backslash\left\{M_{0,2 m}\right\}$, for $n=$ $2 m+1 \equiv 3(4)$. Indeed, in both cases, we have that $d_{\rho, \mu}^{ \pm}\left(\Gamma_{j, h}, \varepsilon_{1}\right)=2^{m-2} d_{\rho}\left|\Lambda_{\varepsilon_{1}, \mu}\right|$, by Theorem 3.2. These manifolds do not have nontrivial harmonic spinors.

(ii) Note that, for every $t$, all $M_{t, 0}, M_{t-1,1}, \ldots, M_{0, t}$ have the same first Betti number. We recall from $\mathrm{MP}$, Proposition 4.1 , that for $1 \leq p \leq n$

$$
\beta_{p}\left(M_{j, h}\right)=\sum_{i=0}^{\left[\frac{p}{2}\right]}\left(\begin{array}{c}
j+h \\
2 i
\end{array}\right)\left(\begin{array}{c}
j+l \\
p-2 i
\end{array}\right) .
$$

Hence, if $\beta_{1}\left(M_{j, h}\right)=\beta_{1}\left(M_{j^{\prime}, h^{\prime}}\right)$, then $\beta_{p}\left(M_{j, h}\right)=\beta_{p}\left(M_{j^{\prime}, h^{\prime}}\right)$ for any $p \geq 1$.

Now, take

$$
\mathcal{F}_{t}=\left\{\left(M_{j, h}, \varepsilon\right): M_{j, h} \in \mathcal{F}^{+} \text {and } j+h=t\right\}
$$

for some fixed $t$ even and $\varepsilon$ as in (4.13). In this way $\mathcal{F}_{t}$ is a family of $t+1$ spin $\mathbb{Z}_{2}$-manifolds which are Dirac isospectral to each other, all having the same $p$-Betti 
numbers for all $1 \leq p \leq n$. Moreover, if we take $n=2 t$, then all $M_{j, h} \in \mathcal{F}_{t}$ are $p$-isospectral for any $p$ odd, by the comments after (4.8).

Example 4.4. Here we give a simple pair of nonhomeomorphic spin $\mathbb{Z}_{2}^{2}$-manifolds that are (twisted) spinor Laplacian isospectral but not (twisted) Dirac isospectral. Let $\Lambda$ be the canonical lattice in $\mathbb{R}^{7}$ and take the Bieberbach groups

$$
\Gamma=\left\langle B_{1} L_{b_{1}}, B_{2} L_{b_{2}}, \Lambda\right\rangle, \quad \Gamma^{\prime}=\left\langle B_{1} L_{b_{1}^{\prime}}, B_{2} L_{b_{2}^{\prime}}, \Lambda\right\rangle,
$$

where $B_{1}=\operatorname{diag}(-1,-1,-1,-1,-1,-1,1), B_{2}=\operatorname{diag}(-1,-1,1,1,1,1,1), B_{1}^{\prime}=$ $\operatorname{diag}(-1,-1,-1,-1,1,1,1), B_{2}^{\prime}=\operatorname{diag}(1,1,-1,-1,-1,-1,1)$, and $b_{1}=\frac{e_{7}}{2}, b_{2}=$ $\frac{e_{1}+e_{3}+e_{7}}{2}, b_{1}^{\prime}=\frac{e_{7}}{2}, b_{2}^{\prime}=\frac{e_{2}}{2}$ are in $\frac{1}{2} \Lambda$. Let $M_{\Gamma}=\Gamma \backslash \mathbb{R}^{7}, M_{\Gamma^{\prime}}=\Gamma^{\prime} \backslash \mathbb{R}^{7}$ be the associated $\mathbb{Z}_{2}^{2}$-manifolds.

By Theorem 2.1 in $\mathrm{MP}$, one can check that $M_{\Gamma}$ and $M_{\Gamma^{\prime}}$, respectively, admit spin structures $\varepsilon, \varepsilon^{\prime}$ with characters $\delta_{\varepsilon}=\left(1, \delta_{2}, 1, \delta_{4}, \delta_{5}, \delta_{6},-1\right)$ and $\delta_{\varepsilon^{\prime}}=$ $\left(\delta_{1}^{\prime}, 1, \delta_{3}^{\prime}, \delta_{4}^{\prime}, \delta_{5}^{\prime}, \delta_{6}^{\prime}, 1\right), \delta_{i}, \delta_{i}^{\prime} \in\{ \pm 1\}$.

Now, $F_{1}(\Gamma)=\left\{B_{1}\right\}$. If we take $\delta_{\varepsilon}=(1,1,1,1,1,1,-1)$, and if $\chi_{\rho}\left(B_{1}\right) \neq 0$, then $\left(M_{\Gamma}, \varepsilon\right)$ has asymmetric Dirac spectrum. Thus, if $\mu_{j}=j+\frac{1}{2}, j \in \mathbb{N}_{0}$, the multiplicity of $\pm 2 \pi \mu$ is given by

$$
d_{\rho, \mu}^{ \pm}(\Gamma, \varepsilon)= \begin{cases}d_{\rho}\left|\Lambda_{\varepsilon, \mu_{j}}\right| \pm 2(-1)^{j} \sigma_{\gamma_{1}} \chi_{\rho}\left(\gamma_{1}\right), & \mu=\mu_{j}, \\ d_{\rho}\left|\Lambda_{\varepsilon, \mu}\right|, & \mu \neq \mu_{j} .\end{cases}
$$

On the other hand $F_{1}\left(\Gamma^{\prime}\right)=\emptyset$. Thus, $M_{\Gamma^{\prime}}$ has a symmetric Dirac spectrum with $d_{\rho, \mu}^{ \pm}\left(\Gamma^{\prime}, \varepsilon^{\prime}\right)=d_{\rho}\left|\Lambda_{\varepsilon^{\prime}, \mu}\right|$.

Now take $\delta_{\varepsilon^{\prime}}=(-1,1,1,1,1,1,1)$. Then $\left(M_{\Gamma}, \varepsilon\right)$ and $\left(M_{\Gamma^{\prime}}, \varepsilon^{\prime}\right)$ are $\Delta_{s, \rho^{-} \text {-isospec- }}$ tral. Indeed, $d_{0}(\Gamma, \varepsilon)=d_{0}\left(\Gamma^{\prime}, \varepsilon^{\prime}\right)=0$ and since $\left|J_{\varepsilon}^{-}\right|=\left|J_{\varepsilon^{\prime}}^{-}\right|=1$, for any $\mu>0$, by Remark 2.2 we have

$$
d_{\rho, \mu}(\Gamma, \varepsilon)=2 d_{\rho}\left|\Lambda_{\varepsilon, \mu}\right|=2 d_{\rho}\left|\Lambda_{\varepsilon^{\prime}, \mu}\right|=d_{\rho, \mu}\left(\Gamma^{\prime}, \varepsilon^{\prime}\right) .
$$

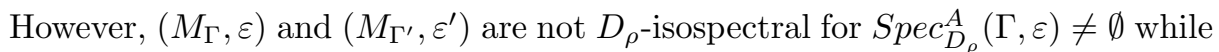
$\operatorname{Spec}_{D_{\rho}}^{A}\left(\Gamma^{\prime}, \varepsilon^{\prime}\right)=\emptyset$. Note that $\operatorname{Spec}_{D_{\rho}}^{S}(\Gamma, \varepsilon)=\operatorname{Spec}_{D_{\rho}}^{S}\left(\Gamma^{\prime}, \varepsilon^{\prime}\right)$.

Example 4.5. Here, we shall give two pairs of $\Delta_{p}$-isospectral for $0 \leq p \leq n$ and $L$-isospectral 4-dimensional $\mathbb{Z}_{2}^{2}$-manifolds $M, M^{\prime}$, one pair being $[L]$-isospectral and the other not. These pairs will be twisted Dirac isospectral, or not, depending on the choices of the spin structures.

Consider the manifolds $M_{i}, M_{i}^{\prime}, 1 \leq i \leq 2$, where $M_{i}=\Gamma_{i} \backslash \mathbb{R}^{4}, M_{i}^{\prime}=\Gamma_{i}^{\prime} \backslash \mathbb{R}^{4}$ and $\Gamma_{i}=\left\langle\gamma_{1}, \gamma_{2}, \Lambda\right\rangle, \Gamma_{i}^{\prime}=\left\langle\gamma_{1}^{\prime}, \gamma_{2}^{\prime}, \Lambda\right\rangle$ are as given in Table 2, where $\gamma_{i}=B_{i} L_{b_{i}}, \gamma_{i}^{\prime}=$ $B_{i} L_{b_{i}^{\prime}}, i=1,2, B_{3}=B_{1} B_{2}, b_{3}=B_{2} b_{1}+b_{2}, b_{3}^{\prime}=B_{2}^{\prime} b_{1}^{\prime}+b_{2}^{\prime}$, and $\Lambda=\mathbb{Z} e_{1} \oplus \cdots \oplus \mathbb{Z} e_{4}$ is the canonical lattice. Furthermore, we take $B_{i}=B_{i}^{\prime}$. In all cases the matrices $B_{i}$ are diagonal and are written as column vectors. We also indicate the translation vectors $b_{i}, b_{i}^{\prime}$ as column vectors, leaving out the coordinates that are equal to zero. We will also use the pair $\tilde{M}_{2}, \tilde{M}_{2}^{\prime}$ of $\mathbb{Z}_{2}^{2}$-manifolds of dimension 6 obtained from the pair $M_{2}, M_{2}^{\prime}$ by adjoining the characters $(-1,1,-1)$ and $(1,-1,-1)$ to $B_{i}$, $1 \leq i \leq 3$, and keeping $b_{i}, b_{i}^{\prime}$ unchanged.

Note that $M_{1}, M_{1}^{\prime}$ and $\tilde{M}_{2}, \tilde{M}_{2}^{\prime}$ are orientable while $M_{2}, M_{2}^{\prime}$ are not.

(i) By Example 3.3 in MR5, the manifolds $M_{1}, M_{1}^{\prime}$ are Sunada isospectral (hence $p$-isospectral for $0 \leq p \leq n$ ) and $[L]$-isospectral.

By Theorem 2.1 in [MP], one can check that $M_{1}$ admits $2^{4}$ spin structures $\varepsilon_{1}$ of the form $\varepsilon_{1}=\left(\delta_{1},-1, \delta_{3},-1 ; \sigma_{1} e_{1} e_{2}, \sigma_{2} e_{2} e_{3}\right)$, and $M_{1}^{\prime}$ carries $2^{3}$ spin structures 
TABLE 2 .

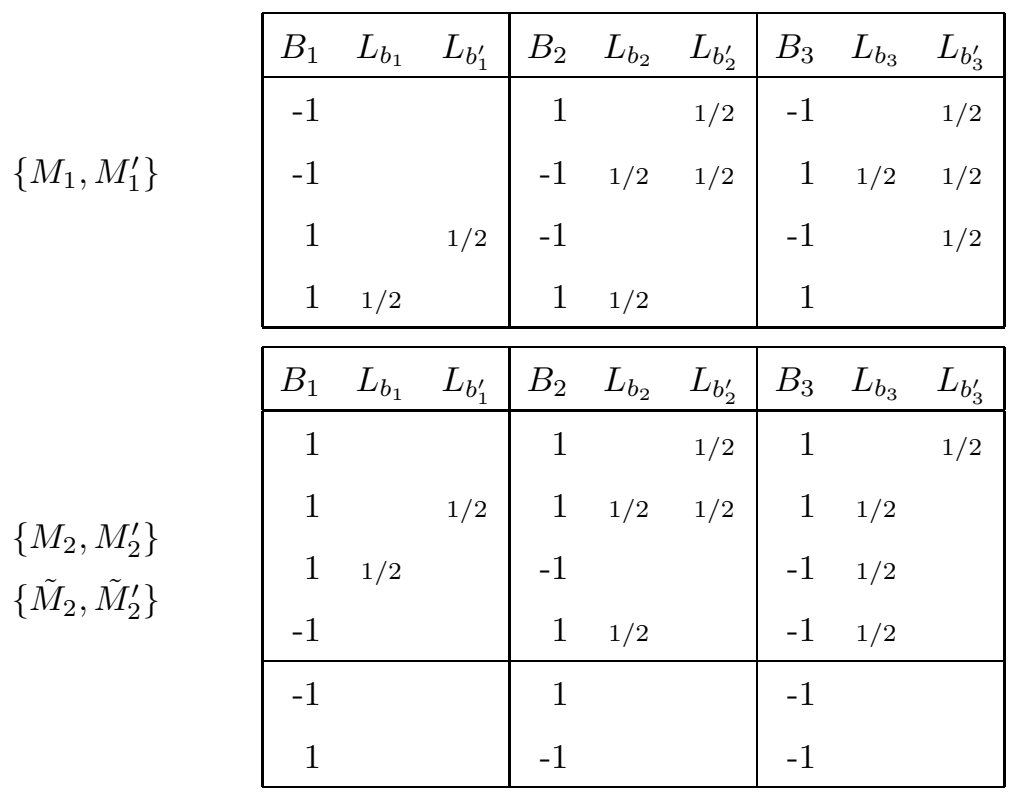

$\varepsilon_{1}^{\prime}$ of the form $\varepsilon_{1}^{\prime}=\left(-1,-1,-1, \delta_{4}^{\prime}, \sigma_{1}^{\prime} e_{1} e_{2}, \sigma_{2}^{\prime} e_{2} e_{3}\right)$, where $\delta_{1}, \delta_{3}, \delta_{4}^{\prime}, \sigma_{1}, \sigma_{2}, \sigma_{1}^{\prime}, \sigma_{2}^{\prime} \in$ $\{ \pm 1\}$.

Since $F_{1}\left(\Gamma_{1}\right)=F_{1}\left(\Gamma_{1}^{\prime}\right)=\emptyset$, by (3.4) we have that $d_{\rho, \mu}^{ \pm}\left(\Gamma_{1}, \varepsilon_{1}\right)=d_{\rho}\left|\Lambda_{\varepsilon_{1}, \mu}\right|$ and $d_{\rho, \mu}^{ \pm}\left(\Gamma_{1}^{\prime}, \varepsilon_{1}^{\prime}\right)=d_{\rho}\left|\Lambda_{\varepsilon_{1}^{\prime}, \mu}\right|$.

Now, if we take $\delta_{\varepsilon_{1}}=(1,-1,1,-1)$, we see that $\left(M_{1}, \varepsilon_{1}\right)$ is not twisted Dirac isospectral to $\left(M_{1}^{\prime}, \varepsilon_{1}^{\prime}\right)$ for any $\varepsilon_{1}^{\prime}$ because $\left|J_{\varepsilon_{1}}^{-}\right|=2$ while $\left|J_{\varepsilon_{1}^{\prime}}^{-}\right| \geq 3$ (see Remark 2.2). However, if we take $\varepsilon_{1}, \varepsilon_{1}^{\prime}$ such that $\delta_{\varepsilon_{1}}=\delta_{\varepsilon_{1}^{\prime}}=(-1,-1,-1,-1)$, then $\left(M_{1}, \varepsilon_{1}\right)$ and $\left(M_{1}^{\prime}, \varepsilon_{1}^{\prime}\right)$ are $D_{\rho}$-isospectral to each other.

(ii) By Example 3.4 in [MR5, the manifolds $M_{2}, M_{2}^{\prime}$ are Sunada isospectral (hence $p$-isospectral for $0 \leq p \leq n$ and $L$-isospectral) but not $[L]$-isospectral. In order to have orientable manifolds we add to $M_{2}, M_{2}^{\prime}$ the characters $(-1,1,-1)$ and $(1,-1,-1)$. The pair $\tilde{M}_{2}, \tilde{M}_{2}^{\prime}$ obtained has the same spectral properties as $M_{2}, M_{2}^{\prime}$. This can be seen by proceeding as in Example 3.4 in MR5.

Again, by Theorem 2.1 in $\mathrm{MP}$, we can check that $\tilde{M}_{2}$ has $2^{5}$ spin structures, $\varepsilon_{2}$, with characters $\delta_{\varepsilon_{2}}=\left(\delta_{1}, 1,-1,-1, \delta_{5}, \delta_{6}\right), \delta_{1}, \delta_{5}, \delta_{6} \in\{ \pm 1\}$; and $\tilde{M}_{2}^{\prime}$ has $2^{6}$ spin structures, $\varepsilon_{2}^{\prime}$, with characters $\delta_{\varepsilon_{2}^{\prime}}=\left(1,-1, \delta_{3}^{\prime}, \delta_{4}^{\prime}, \delta_{5}^{\prime}, \delta_{6}^{\prime}\right)$, where $\delta_{3}^{\prime}, \delta_{4}^{\prime}, \delta_{5}^{\prime}, \delta_{6}^{\prime} \in$ $\{ \pm 1\}$.

Now, $d_{\rho, \mu}^{ \pm}\left(\tilde{\Gamma}_{2}, \varepsilon_{2}\right)=d_{\rho}\left|\Lambda_{\varepsilon_{2}, \mu}\right|$ and $d_{\rho, \mu}^{ \pm}\left(\tilde{\Gamma}_{2}^{\prime}, \varepsilon_{2}^{\prime}\right)=d_{\rho}\left|\Lambda_{\varepsilon_{2}^{\prime}, \mu}\right|$. As in (i), if we take spin structures $\varepsilon_{2}, \varepsilon_{2}^{\prime}$ with $\left|J_{\varepsilon_{2}}^{-}\right|=\left|J_{\varepsilon_{2}^{\prime}}^{-}\right|$, we see that $\left(\tilde{M}_{2}, \varepsilon_{2}\right)$ and $\left(\tilde{M}_{2}^{\prime}, \varepsilon_{2}^{\prime}\right)$ are $D_{\rho^{-}}$ isospectral to each other, whereas if we take $\varepsilon_{2}, \varepsilon_{2}^{\prime}$ such that $\left|J_{\varepsilon_{2}}^{-}\right| \neq\left|J_{\varepsilon_{2}^{\prime}}^{-}\right|$, then $\left(\tilde{M}_{2}, \varepsilon_{2}\right)$ and $\left(\tilde{M}_{2}^{\prime}, \varepsilon_{2}^{\prime}\right)$ are not $D_{\rho}$-isospectral.

Example 4.6. We now construct a large family of pairwise nonhomeomorphic twisted Dirac isospectral flat $2 n$-manifolds, with holonomy group $\mathbb{Z}_{2}^{n-1}$. We will apply the doubling procedure in [JR] or in BDM] to the family of Hantzsche-Wendt manifolds (see $[\mathrm{MR}]$ ). 
We first recall some facts from $\mathrm{MR}$. Let $n$ be odd. A Hantzsche-Wendt group (or $H W$ group) is an $n$-dimensional orientable Bieberbach group $\Gamma$ with holonomy group $F \simeq \mathbb{Z}_{2}^{n-1}$ such that the action of every $B \in F$ diagonalizes on the canonical $\mathbb{Z}$-basis $e_{1}, \ldots, e_{n}$ of $\Lambda$. The holonomy group $F$ can thus be identified to the diagonal subgroup $\left\{B: B e_{i}= \pm e_{i}, 1 \leq i \leq n\right.$, $\left.\operatorname{det} B=1\right\}$, and $M_{\Gamma}=\Gamma \backslash \mathbb{R}^{n}$ is called a Hantzsche-Wendt (or $H W$ ) manifold.

We denote by $B_{i}$ the diagonal matrix fixing $e_{i}$ and such that $B_{i} e_{j}=-e_{j}$ (if $j \neq i$ ), for each $1 \leq i \leq n$. Clearly, $F$ is generated by $B_{1}, B_{2}, \ldots, B_{n-1}$ and furthermore $B_{n}=B_{1} B_{2} \ldots B_{n-1}$.

Any HW group has the form $\Gamma=\left\langle\gamma_{1}, \ldots, \gamma_{n-1}, L_{\lambda}: \lambda \in \Lambda\right\rangle$, where $\gamma_{i}=B_{i} L_{b_{i}}$ for some $b_{i} \in \mathbb{R}^{n}, 1 \leq i \leq n-1$, and where it may be assumed that the components $b_{i j}$ of $b_{i}$ satisfy $b_{i j} \in\left\{0, \frac{1}{2}\right\}$, for $1 \leq i, j \leq n$. Also, since $\left(\Lambda^{p}\left(\mathbb{R}^{n}\right)\right)^{\mathrm{F}}=0$ for any $1 \leq p \leq n-1$, it follows that all HW manifolds are rational homology spheres. We further recall that, as shown in [MR (by using a rather small subfamily $\mathcal{H}_{1}$ ), the cardinality $h_{n}$ of the family of all HW groups under isomorphism satisfies $h_{n}>\frac{2^{n-3}}{n-1}$. Moreover, the cardinality of the set of Laplace isospectral, pairwise nonisomorphic, pairs of HW groups grows exponentially with $n$.

Relative to the spin condition, it is easy to verify using $\left(\varepsilon_{1}\right)$ in [MP], (2.3), that the manifolds in this family are generally not spin. Indeed, we now show that no HW manifold with $n=4 k+1$ is spin. We note that $\gamma_{k}^{2}=L_{e_{k}}$ for each $1 \leq k \leq n$. Hence a spin structure $\varepsilon$ must satisfy $\delta_{k}=\varepsilon\left(L_{e_{k}}\right)=\varepsilon\left(\gamma_{k}^{2}\right)=\varepsilon\left(\gamma_{k}\right)^{2}=$ $\left( \pm e_{1} \ldots e_{k-1} e_{k+1} \ldots e_{n}\right)^{2}=1$, since $n=4 k+1$. Thus, it follows that $\varepsilon_{\mid \Lambda}=I d$. On the other hand $\left(\gamma_{i} \gamma_{j}\right)^{2}=L_{\lambda}$ for some $\lambda \neq 0$ and since $\varepsilon\left(\gamma_{i} \gamma_{j}\right)= \pm e_{i} e_{j}$, it follows that $\varepsilon\left(L_{\lambda}\right)=\varepsilon\left( \pm e_{i} e_{j}\right)^{2}=-1$, a contradiction.

In the case $n=4 k+3, k>0$, we shall show that no HW manifold in the family $\mathcal{H}_{1}$ (see $[\mathrm{MR}$ ) is spin. More generally, assume that there are three consecutive generators of $\Gamma, \gamma_{i}=B_{i} L_{b_{i}}$ with $b_{i}=L_{\left(e_{i}+e_{i-1}\right) / 2}, i \geq 2$. Thus, if $\gamma:=\gamma_{i} \gamma_{i+1} \gamma_{i+2}=B_{i} B_{i+1} B_{i+2} L_{\left(e_{i-1}+e_{i+2}\right) / 2}$, then we have $\gamma^{2}=\gamma_{i+2}^{2}=L_{e_{i+2}}$. This gives a contradiction since $\varepsilon(\gamma)^{2}=1$ (the multiplicity of the eigenvalue -1 for $B_{i} B_{i+1} B_{i+2}$ is $4 k$ ) while $\varepsilon\left(\gamma_{i+2}\right)^{2}=-1$ (the multiplicity of the eigenvalue -1 for $B_{i+2}$ is $4 k+2$ ). Actually, J.P. Rossetti has recently shown to us a proof (that still uses the criterion in $\mathrm{MP}$ ) that no HW manifold can admit a spin structure.

We will now consider for any HW group $\Gamma$, the group $\mathrm{d} \Gamma$, defined by the doubling construction in [JR] or [BDM], namely $\mathrm{d} \Gamma=\left\langle d B L_{(b, b)}, L_{\left(\lambda_{1}, \lambda_{2}\right)}: B L_{b} \in \Gamma, \lambda_{1}, \lambda_{2} \in\right.$ $\Lambda\rangle$, where $d B:=\left[\begin{array}{cc}B & 0 \\ 0 & B\end{array}\right]$. This yields a Bieberbach group of dimension $2 n$, with the same holonomy group $\mathbb{Z}_{2}^{n-1}$ as $\Gamma$, and with the additional property that the associated manifold $M_{\mathrm{d} \Gamma}$ is Kähler. The reason why we use $\mathrm{d} \Gamma$ in place of $\Gamma$ is that $M_{\mathrm{d} \Gamma}$ is always spin (see MP, Remark 2.4).

We will need the following facts:

(i) If $\Gamma$ is a $H W$ group, then $M_{\mathrm{d} \Gamma}$ admits $2^{n-1}$ spin structures of trivial type.

(ii) If $\Gamma$ runs through all $H W$ groups, all manifolds $M_{\mathrm{d} \Gamma}$ endowed with spin structures of trivial type are twisted Dirac isospectral to each other.

(iii) If $\Gamma, \Gamma^{\prime}$ are nonisomorphic $H W$ groups, then $\mathrm{d} \Gamma, \mathrm{d} \Gamma^{\prime}$ are nonisomorphic Bieberbach groups.

(iv) Two $H W$ groups $\Gamma, \Gamma^{\prime}$ are Laplace isospectral if and only if $\mathrm{d} \Gamma$ and $\mathrm{d} \Gamma^{\prime}$ are Laplace isospectral.

Now, (i) and (ii) are direct consequences of Remark 2.4 in [MP] and of (i) of Theorem 3.2, respectively. 
Proof of (iii). This assertion follows by an argument very similar to that given in the proof of Proposition 1.5 in $\mathrm{MR}$. We shall only sketch it. An isomorphism between $\mathrm{d} \Gamma$ and $\mathrm{d} \Gamma^{\prime}$ must be given by conjugation by $C L_{c}$ with $C \in \mathrm{GL}(2 n, \mathbb{R})$ and $c \in \mathbb{R}^{2 n}$. Now $C(\Lambda \oplus \Lambda)=\Lambda \oplus \Lambda$ implies $C \in \mathrm{GL}(2 n, \mathbb{Z})$ and furthermore for each $1 \leq i \leq n-1$,

$$
C L_{c} d B_{i} L_{\left(b_{i}, b_{i}\right)} L_{-c} C^{-1}=d B_{\sigma(i)} L_{\left(b_{\sigma(i)}^{\prime}, b_{\sigma(i)}^{\prime}\right)}
$$

where $\sigma \in S_{n}$. Thus $C d B_{i} C^{-1} L_{C\left(\left(b_{i}, b_{i}\right)+\left(d B_{i}-I d\right) c\right)}$. In particular, this implies that $C d B_{i} C^{-1}=d B_{\sigma(i)}$ for each $1 \leq i \leq n$, with $\sigma \in S_{n}$. Thus, there is an $n \times n$ permutation matrix $P$ such that $D:=C d P C^{-1}$ commutes with $d B_{i}$ for each $i$, thus $D$ preserves $\mathbb{Z} e_{i} \oplus \mathbb{Z} e_{n+i}$ for each $i$. It is easy to see that conjugation by such $D$ yields an automorphism of $\mathrm{d} \Gamma^{\prime}$. Thus, conjugation by $D C L_{c}=d P L_{c}$ takes $\mathrm{d} \Gamma$ onto $\mathrm{d} \Gamma^{\prime}$ isomorphically and furthermore

$$
\begin{aligned}
d P L_{c} d B_{i} L_{\left(b_{i}, b_{i}\right)} L_{-c} d P^{-1} & =d\left(P B_{i} P\right)^{-1} L_{d P\left(\left(b_{i}, b_{i}\right)+\left(d B_{i}-I d\right) c\right)} \\
& =d B_{\sigma(i)} L_{\left(b_{i}^{\prime}, b_{i}^{\prime}\right)} .
\end{aligned}
$$

This implies that $c=\left(c_{1}, c_{1}\right)$, $\bmod \Lambda$, with $c_{1} \in \frac{1}{4} \Lambda$ and hence, conjugation by $P L_{c_{1}}$ gives an isomorphism between $\Gamma$ and $\Gamma^{\prime}$, a contradiction.

Proof of (iv). Since HW groups are of diagonal type, then $\Gamma, \Gamma^{\prime}$ are Laplace isospectral if and only if they are Sunada isospectral, that is, if they have the same Sunada numbers (see [MR3, MR4]). We claim that this is the case if and only if $\mathrm{d} \Gamma$ and $\mathrm{d} \Gamma^{\prime}$ are Sunada isospectral to each other.

Indeed, we recall that for $0 \leq t \leq s \leq n$, and $\Gamma$ of diagonal type, the Sunada numbers of $\Gamma$ are defined by

$$
c_{d, t}(\Gamma):=\mid\left\{B L_{b} \in \Gamma: n_{B}=d \text { and } n_{B}\left(\frac{1}{2}\right)=t\right\} \mid,
$$

where, for $B L_{b} \in \Gamma, n_{B}:=\operatorname{dim}\left(\mathbb{R}^{n}\right)^{B}=\left|\left\{1 \leq i \leq n: B e_{i}=e_{i}\right\}\right|$ and $n_{B}\left(\frac{1}{2}\right):=$ $\mid\left\{1 \leq i \leq n: B e_{i}=e_{i}\right.$ and $\left.b \cdot e_{i} \equiv \frac{1}{2} \bmod (\mathbb{Z})\right\} \mid$. Now, it is clear from the definitions that $c_{2 s, 2 t}(\mathrm{~d} \Gamma)=c_{s, t}(\Gamma)$ for each $0 \leq t \leq s \leq n$ and $c_{u, v}(\mathrm{~d} \Gamma)=0$, if either $u$ or $v$ is odd. This clearly implies that $\Gamma$ and $\Gamma^{\prime}$ have the same Sunada numbers if and only if $\mathrm{d} \Gamma$ and $\mathrm{d} \Gamma^{\prime}$ do, that is, if and only if $\mathrm{d} \Gamma$ and $\mathrm{d} \Gamma$ are Sunada-isospectral to each other.

Thus, for each $n$ odd, by (i), (ii), (iii), (iv), the above construction yields a family of cardinality that depends exponentially on $n$, of Kähler flat manifolds of dimension $2 n$, all pairwise nonhomeomorphic and all twisted Dirac isospectral to each other, having only $2 d_{\rho}$ harmonic spinors for every trivial spin structure chosen (see (iii) of Theorem 3.2). Within this family, by (iv) and [MR], there are exponentially many pairs that are Sunada isospectral, hence $p$-isospectral for all $p$. However, generically, two such manifolds will not be $p$-isospectral for any value of $p$ (see for instance [MR2] in the case $n=7$ ).

We note that if we repeat the doubling procedure, then the set of all $M_{\mathrm{d}^{2} \Gamma}$, with $\Gamma$ a HW group, is an exponential family of hyperkähler manifolds with the same spectral properties as the family $M_{\mathrm{d} \Gamma}$, but now having $2^{n+1} d_{\rho}$ harmonic spinors for every spin structure of trivial type chosen.

Remark 4.7. If one looks at the family of all flat manifolds with holonomy group $\mathbb{Z}_{2}^{n-1}$ (see [RS]) for $n=4 r+3$, then it is shown in [MPR that a subfamily of this family has cardinality $2^{\frac{(n-1)(n-2)}{2}}$. If we apply the doubling procedure to this 
family, one shows that considerations (i),...,(iv) in Example 4.6 remain valid, hence one obtains a family of twisted Dirac isospectral, pairwise nonhomeomorphic, $2 n$-manifolds of cardinality $2 \frac{(n-1)(n-2)}{2}$.

\section{ETA INVARIANTS OF $\mathbb{Z}_{p}$-MANIFOLDS}

In this section we shall illustrate the results in Section 2 by using the expression (2.18) of the eta series, to compute explicitly the eta invariant of certain flat $p$ manifolds with cyclic holonomy group $\mathbb{Z}_{p}, p=4 r+3, p$ prime, for the two existing spin structures. In $\mathrm{SS}$, the authors give an expression for the eta invariant and harmonic spinors of this family (without assuming $p$ to be prime) in terms of the solutions of certain equations in congruences. They give explicit values for $p=3,7$. Here we shall give an explicit expression for the eta invariant of this family in terms of Legendre symbols and special values of trigonometric functions. At the end we give a table with the values of $\eta$ for any prime $p \leq 503$. For $n=3$ they coincide with those computed in [Pf] (and [SS]). Our formulas for the eta series involve trigonometric sums and resemble those obtained in $[\mathrm{HZ}$ to compute the $G$-index of elliptic operators for certain low-dimensional manifolds.

We thus assume that $F$ is cyclic of order $p=4 r+3$ (i.e. $m=2 r+1$ ) with $p$ prime. Let $\Gamma=\left\langle B L_{b}, L_{\Lambda}\right\rangle$. Here $\Lambda=\sum_{i=1}^{p} \mathbb{Z} e_{i}, B$ is of order $p$ with $B\left(e_{i}\right)=e_{i+1}$ for $1 \leq i \leq p-2, B\left(e_{p-1}\right)=-\sum_{i=1}^{p-1} e_{i}, B\left(e_{p}\right)=e_{p}$ and $b=\frac{1}{p} e_{p}$.

Now, there exists $D \in \mathrm{GL}_{p}(\mathbb{R}), D e_{p}=e_{p}$ such that $C:=D B D^{-1} \in \mathrm{SO}(p)$. Thus, $\Gamma_{p}:=D \Gamma D^{-1}=\left\langle\gamma=C L_{\frac{e_{p}}{p}}, D \Lambda\right\rangle$ is a Bieberbach group and $M_{p}=\Gamma_{p} \backslash \mathbb{R}^{p}$ is an orientable $\mathbb{Z}_{p}$-manifold of dimension $p$. The vectors $f_{i}:=D e_{i}$ for $1 \leq i \leq p-1$, and $f_{p}=e_{p}$ give a $\mathbb{Z}$-basis of $D \Lambda$.

Since the eigenvalues of $B$ and $C$ are the $p$-roots of unity: $\left\{e^{\frac{2 \pi i k}{p}}: 1 \leq k \leq p-1\right\}$, we can then assume by further conjugation in $\mathrm{SO}(p)$ that $C=x_{0}\left(\frac{2 \pi}{p}, \ldots, \frac{2 m \pi}{p}\right)$ (see (1.4)) with $m=\frac{p-1}{2}$.

We now note that Theorem 2.1 in [MP], stated for $\mathbb{Z}_{2}^{k}$-manifolds, also holds for $\mathbb{Z}_{n}$-manifolds, replacing condition $\left(\varepsilon_{1}\right): \varepsilon\left(\gamma^{2}\right)=\varepsilon(\gamma)^{2}$ by $\left(\varepsilon_{1}^{\prime}\right): \varepsilon\left(\gamma^{n}\right)=u_{B}^{n}$ for any $u_{B} \in \operatorname{Spin}(n)$ such that $\mu\left(u_{B}\right)=B$, and keeping condition $\left(\varepsilon_{2}\right)$ in MP, i.e. $\varepsilon\left(L_{(B-I d)(\lambda)}\right)=1$ for any $\lambda \in \Lambda$. Thus, using conditions $\left(\varepsilon_{1}^{\prime}\right)$ and $\left(\varepsilon_{2}\right)$ one can see that $M_{p}:=\Gamma_{p} \backslash \mathbb{R}^{p}$ has exactly two spin structures, $\varepsilon_{1}, \varepsilon_{2}$, given on the generators of $\Gamma$ by

$$
\begin{gathered}
\varepsilon_{1}\left(L_{f_{j}}\right)=\varepsilon_{2}\left(L_{f_{j}}\right)=1(1 \leq j \leq p-1), \quad \varepsilon_{1}\left(L_{e_{p}}\right)=1, \varepsilon_{2}\left(L_{e_{p}}\right)=-1, \\
\varepsilon_{1}(\gamma)=(-1)^{r+1} x\left(\frac{\pi}{p}, \frac{2 \pi}{p}, \ldots, \frac{(2 r+1) \pi}{p}\right), \quad \varepsilon_{2}(\gamma)=(-1)^{r} x\left(\frac{\pi}{p}, \frac{2 \pi}{p}, \ldots, \frac{(2 r+1) \pi}{p}\right),
\end{gathered}
$$

in the notation of (1.4). We can now state:

Theorem 5.1. Let $p=4 r+3$ be a prime and let $\Gamma_{p}, \varepsilon_{1}, \varepsilon_{2}$ be as above. Then, the eta series of $M_{p}=\Gamma_{p} \backslash \mathbb{R}^{p}$, for $\varepsilon_{1}$ and $\varepsilon_{2}$ are respectively given by:

$$
\begin{aligned}
& \eta_{\left(\Gamma_{p}, \rho, \varepsilon_{1}\right)}(s)=\frac{-2}{\sqrt{p}(2 \pi p)^{s}} \sum_{k=1}^{p-1} \chi_{\rho}\left(\gamma^{k}\right)\left(\frac{k}{p}\right) \sum_{l=1}^{p-1} \sin \left(\frac{2 l \pi k}{p}\right) \zeta\left(s, \frac{l}{p}\right), \\
& \eta_{\left(\Gamma_{p}, \rho, \varepsilon_{2}\right)}(s)=\frac{-2}{\sqrt{p}(2 \pi p)^{s}} \sum_{k=1}^{p-1} \chi_{\rho}\left(\gamma^{k}\right)(-1)^{k}\left(\frac{k}{p}\right) \sum_{l=0}^{p-1} \sin \left(\frac{(2 l+1) \pi k}{p}\right) \zeta\left(s, \frac{2 l+1}{2 p}\right),
\end{aligned}
$$


and the eta invariants have the expressions

$$
\begin{aligned}
& \eta_{\rho, \varepsilon_{1}}=\frac{-2}{\sqrt{p}} \sum_{k=1}^{\frac{p-1}{2}} \chi_{\rho}\left(\gamma^{k}\right)\left(\frac{k}{p}\right) \cot \left(\frac{k \pi}{p}\right), \\
& \eta_{\rho, \varepsilon_{2}}=\frac{-2}{\sqrt{p}} \sum_{k=1}^{\frac{p-1}{2}} \chi_{\rho}\left(\gamma^{k}\right)(-1)^{k}\left(\frac{k}{p}\right) \operatorname{cosec}\left(\frac{k \pi}{p}\right),
\end{aligned}
$$

where $(\dot{\bar{p}})$ denotes the Legendre symbol.

Note. The theorem shows that one can have a spin structure of trivial type (namely $\varepsilon_{1}$ ) with asymmetric Dirac spectrum, in contrast with the situation in the case of holonomy group $\mathbb{Z}_{2}^{k}$.

Expressions (5.1) and (5.2) can be simplified further by using identities in number theory. In particular one shows that the eta invariants take integer values. We plan to get deeper into this question in a sequel to this paper.

Proof. We will compute the different ingredients in formula (2.18) for the eta function for the two given spin structures. For $1 \leq k \leq p-1$ we have $\left(\Lambda_{\varepsilon}^{*}\right)^{B^{k}}=\mathbb{Z} e_{p}$ for $\varepsilon_{1}$, while $\left(\Lambda_{\varepsilon}^{*}\right)^{B^{k}}=\left(\mathbb{Z}+\frac{1}{2}\right) e_{p}$ for $\varepsilon_{2}$. In both cases $\left(\Lambda_{\varepsilon, \mu}^{*}\right)^{B^{k}}=\left\{ \pm \mu e_{p}\right\}$. Thus, $\mu=j$ for $\varepsilon_{1}$ and $\mu=j-\frac{1}{2}$ for $\varepsilon_{2}$ with $j \in \mathbb{N}$. We take $x_{\gamma^{k}}=\varepsilon_{i}(\gamma)^{k}$ for $i=1,2$. This implies that $\sigma\left(e_{p}, x_{\gamma^{k}}\right)=1$ for $\varepsilon_{1}, \varepsilon_{2}$.

Since $\gamma^{k}=B^{k} L_{\frac{k}{p} e_{p}}$, by (2.7) and Remark 2.4, for $1 \leq k \leq p-1$, we have

$$
e_{\mu, \gamma^{k}, \sigma}\left(\delta_{\varepsilon}\right)=e^{-2 \pi i \frac{\mu k}{p}} \sigma\left(\mu e_{p}, x_{\gamma^{k}}\right)+e^{2 \pi i \frac{\mu k}{p}} \sigma\left(-\mu e_{p}, x_{\gamma^{k}}\right)=-2 i \sin \left(\frac{2 \pi \mu k}{p}\right) .
$$

Thus, up to the factor $-2 i$, the sums over $\mu \in \frac{1}{2 \pi} \mathcal{A}$ corresponding to $\gamma=\gamma^{k}$ in (2.18) for $\varepsilon_{1}$ and $\varepsilon_{2}$ are respectively equal to

$$
\begin{gathered}
\sum_{j=1}^{\infty} \frac{\sin \left(\frac{2 j \pi k}{p}\right)}{j^{s}}=\sum_{l=1}^{p-1} \sin \left(\frac{2 l \pi k}{p}\right) \sum_{j=1}^{\infty} \frac{1}{(p j+l)^{s}}=\frac{1}{p^{s}} \sum_{l=1}^{p-1} \sin \left(\frac{2 l \pi k}{p}\right) \zeta\left(s, \frac{l}{p}\right), \\
\sum_{j=0}^{\infty} \frac{\sin \left(\frac{(2 j+1) \pi k}{p}\right)}{\left(j+\frac{1}{2}\right)^{s}}=\frac{1}{p^{s}} \sum_{l=0}^{p-1} \sin \left(\frac{(2 l+1) \pi k}{p}\right) \zeta\left(s, \frac{2 l+1}{2 p}\right),
\end{gathered}
$$

where $\zeta(s, \alpha)=\sum_{j=0}^{\infty} \frac{1}{(j+\alpha)^{s}}$ denotes the Hurwitz zeta function for $\alpha \in(0,1]$.

We now compute the product of sines in (2.18) in both cases. We note that $t_{j}\left(x_{\gamma^{k}}\right)$ (see (1.4) ) depends on $\gamma^{k}$ and also on $\varepsilon_{1}, \varepsilon_{2}$. We have, for $\varepsilon_{h}, h=1,2$ :

$$
\begin{aligned}
\prod_{j=1}^{\frac{p-1}{2}} \sin t_{j}\left(x_{\gamma^{k}}\right) & =(-1)^{k(r+h)} \prod_{j=1}^{\frac{p-1}{2}} \sin \left(\frac{\pi j k}{p}\right) \\
& =(-1)^{k(r+h)} \prod_{j=1}^{\frac{p-1}{2}}(-1)^{\left[\frac{j k}{p}\right]} \prod_{j=1}^{\frac{p-1}{2}} \sin \left(\frac{\pi j}{p}\right) \\
& =(-1)^{k(r+h)}(-1)^{s_{p}(k)} \frac{\sqrt{p}}{2^{2 r+1}},
\end{aligned}
$$


where we have put $s_{p}(k)=\sum_{j=1}^{\frac{p-1}{2}}\left[\frac{j k}{p}\right]$ and used the identities

$$
\sin (\pi z)=\frac{\pi}{\Gamma(z) \Gamma(1-z)}, \quad(2 \pi)^{\frac{p-1}{2}} \Gamma(z)=p^{z-\frac{1}{2}} \Gamma\left(\frac{z}{p}\right) \Gamma\left(\frac{z+1}{p}\right) \cdots \Gamma\left(\frac{z+p-1}{p}\right) .
$$

Now, if $(\dot{\bar{p}})$ denotes the Legendre symbol, then (see Ap, Theorems 9.6 and 9.7) we have, since $p=4 r+3$,

$$
(-1)^{s_{p}(k)}=(-1)^{(k-1)\left(\frac{p^{2}-1}{8}\right)}\left(\frac{k}{p}\right)=(-1)^{(k-1)(r+1)}\left(\frac{k}{p}\right) .
$$

In this way, we obtain

$$
\prod_{j=1}^{\frac{p-1}{2}} \sin t_{j}\left(x_{\gamma^{k}}\right)= \begin{cases}(-1)^{(r+1)} 2^{-2 r-1} \sqrt{p}\left(\frac{k}{p}\right) & \text { for } \varepsilon_{1}, \\ (-1)^{(r+1)} 2^{-2 r-1}(-1)^{k} \sqrt{p}\left(\frac{k}{p}\right) & \text { for } \varepsilon_{2} .\end{cases}
$$

Now, starting from (2.18) and using (5.3) and (5.4) we finally arrive at the expressions for the $\eta$-series of $\left(M_{p}, \varepsilon_{h}\right), h=1,2$, given in (5.1).

We now compute the eta invariants. Using the fact that $\zeta(0, \alpha)=\frac{1}{2}-\alpha([\mathrm{WW}$, 13.21), together with the fact that $\sum_{l=1}^{p-1} \sin \left(\frac{2 l \pi k}{p}\right)=\sum_{l=0}^{p-1} \sin \left(\frac{(2 l+1) \pi k}{p}\right)=0$ for every $1 \leq k \leq p-1$, we see that the sums over $l$ in the expressions (5.1), when evaluated at $s=0$, are respectively equal to

$$
-\frac{1}{p} \sum_{l=1}^{p-1} l \sin \left(\frac{2 l \pi k}{p}\right), \text { for } \varepsilon_{1}, \quad-\frac{1}{2 p} \sum_{l=0}^{p-1}(2 l+1) \sin \left(\frac{(2 l+1) \pi k}{p}\right) \text {, for } \varepsilon_{2} .
$$

We claim that

$$
\sum_{l=1}^{p-1} l \sin \left(\frac{2 l \pi k}{p}\right)=-\frac{p}{2} \cot \left(\frac{k \pi}{p}\right), \quad \sum_{l=0}^{p-1} l \sin \left(\frac{(2 l+1) \pi k}{p}\right)=-\frac{p}{2} \operatorname{cosec}\left(\frac{k \pi}{p}\right) .
$$

Indeed, by differentiating the identity $\frac{1}{2}+\sum_{l=1}^{p-1} \cos (l x)=\frac{\sin \left(\left(p-\frac{1}{2}\right) x\right)}{2 \sin \left(\frac{x}{2}\right)}$, we get

$$
-\sum_{l=1}^{p-1} l \sin (l x)=\frac{(2 p-1) \cos \left(\left(p-\frac{1}{2}\right) x\right)}{4 \sin \left(\frac{x}{2}\right)}-\frac{\cos \left(\frac{x}{2}\right) \sin \left(\left(p-\frac{1}{2}\right) x\right)}{4 \sin ^{2}\left(\frac{x}{2}\right)} .
$$

Evaluating both sides at $x=\frac{2 k \pi}{p}$ yields the first equality in (5.6).

To verify the second identity we first note that

$$
\sum_{l=1}^{p-1} l \cos \left(\frac{2 l \pi k}{p}\right)=p \sum_{l=1}^{\frac{p-1}{2}} \cos \left(\frac{2 l \pi k}{p}\right)=-\frac{p}{2} .
$$

Using this expression together with the first identity in (5.6) we have

$$
\begin{aligned}
\sum_{l=0}^{p-1} l \sin \left(\frac{(2 l+1) \pi k}{p}\right) & =\cos \left(\frac{\pi k}{p}\right) \cot \left(\frac{\pi k}{p}\right)\left(-\frac{p}{2}\right)+\sin \left(\frac{\pi k}{p}\right)\left(-\frac{p}{2}\right) \\
& =-\frac{p}{2} \operatorname{cosec}\left(\frac{\pi k}{p}\right) .
\end{aligned}
$$

Hence, from (5.1), (5.5) and (5.6) we obtain

$$
\eta_{\varepsilon_{1}}=\frac{-1}{\sqrt{p}} \sum_{k=1}^{p-1}\left(\frac{k}{p}\right) \cot \left(\frac{k \pi}{p}\right), \quad \eta_{\varepsilon_{2}}=\frac{-1}{\sqrt{p}} \sum_{k=1}^{p-1}(-1)^{k}\left(\frac{k}{p}\right) \operatorname{cosec}\left(\frac{k \pi}{p}\right) .
$$


We finally note that the contributions of $k$ and $p-k$ to the above sums are equal to each other, that is,

$$
\begin{aligned}
\left(\frac{k}{p}\right) \cot \left(\frac{k \pi}{p}\right) & =\left(\frac{p-k}{p}\right) \cot \left(\frac{(p-k) \pi}{p}\right) \\
(-1)^{k}\left(\frac{k}{p}\right) \operatorname{cosec}\left(\frac{k \pi}{p}\right) & =(-1)^{p-k}\left(\frac{p-k}{p}\right) \operatorname{cosec}\left(\frac{(p-k) \pi}{p}\right) .
\end{aligned}
$$

These identities can be easily verified using

$$
\left(\frac{p-k}{p}\right)=\left(\frac{-k}{p}\right)=\left(\frac{-1}{p}\right)\left(\frac{k}{p}\right)=(-1)^{\frac{p-1}{2}}\left(\frac{k}{p}\right)=-\left(\frac{k}{p}\right),
$$

where in the last equality we have used $p \equiv 3(4)$.

Taking into account this observation, we obtain expressions (5.2) in the proposition.

We now look at the simplest case when $p=3$ and $\rho=1$, first considered in $[\mathrm{Pf}]$. We have $r=0$ and $\varepsilon_{1}=\left(1,1,1,-x\left(\frac{\pi}{3}\right)\right)=\left(1,1,1, x\left(\frac{\pi}{3}+\pi\right)\right), \varepsilon_{2}=\left(1,1,-1, x\left(\frac{\pi}{3}\right)\right)$ (in the notation of Section 4 ; see (4.1)). Since $\left(\frac{1}{p}\right)=1$ we obtain

$$
\begin{aligned}
& \eta_{\varepsilon_{1}}(0)=\frac{-2}{\sqrt{3}}\left(\left(\frac{1}{3}\right) \cot \left(\frac{\pi}{3}\right)\right)=-\frac{2}{\sqrt{3}} \cdot \frac{1}{\sqrt{3}}=-\frac{2}{3}, \\
& \eta_{\varepsilon_{2}}(0)=\frac{-2}{\sqrt{3}}\left(-\left(\frac{1}{3}\right) \operatorname{cosec}\left(\frac{\pi}{3}\right)\right)=\frac{2}{\sqrt{3}} \cdot \frac{2}{\sqrt{3}}=\frac{4}{3} .
\end{aligned}
$$

It is reassuring to see that the values are coincidental with those in $[\mathrm{Pf}]$, after all these calculations.

To conclude this section, we shall give explicitly the eta invariants for all $p$ manifolds in the family, $p=4 r+3$ prime, $7 \leq p \leq 503$, obtained with the help of a computer, using formulas (5.2), in the case $(\rho, V)=(1, \mathbb{C})$. We also give some values of $d_{0}\left(\varepsilon_{1}\right)$, the dimension of the space of harmonic spinors using (2.10) and (6.5). Note that $d_{0}\left(\varepsilon_{2}\right)=0$ by Theorem 2.5. We summarize the information in the following tables:

\begin{tabular}{|c|c|c|c|r|}
\hline$r$ & $p=4 r+3$ & $\eta_{\varepsilon_{1}}$ & $\eta_{\varepsilon_{2}}$ & $d_{0}\left(\varepsilon_{1}\right)$ \\
\hline 0 & 3 & $-\frac{2}{3}$ & $\frac{4}{3}$ & 0 \\
1 & 7 & -2 & 0 & 2 \\
2 & 11 & -2 & 4 & 2 \\
4 & 19 & -2 & 4 & 26 \\
5 & 23 & -6 & 0 & 90 \\
7 & 31 & -6 & 0 & 1058 \\
10 & 43 & -2 & 4 & 48770 \\
11 & 47 & -10 & 0 & 178482 \\
14 & 59 & -6 & 12 & 9099506 \\
16 & 67 & -2 & 4 & 128207978 \\
17 & 71 & -14 & 0 & 483939978 \\
\hline
\end{tabular}

\begin{tabular}{|c|c|c|c|}
\hline$r$ & $p=4 r+3$ & $\eta_{\varepsilon_{1}}$ & $\eta_{\varepsilon_{2}}$ \\
\hline 19 & 79 & -10 & 0 \\
20 & 83 & -6 & 12 \\
25 & 103 & -10 & 0 \\
26 & 107 & -6 & 12 \\
31 & 127 & -10 & 0 \\
32 & 131 & -10 & 20 \\
34 & 139 & -6 & 12 \\
37 & 151 & -14 & 0 \\
40 & 163 & -2 & 4 \\
41 & 167 & -22 & 0 \\
\hline
\end{tabular}




\begin{tabular}{|c|c|c|c|}
\hline$r$ & $p$ & $\eta_{\varepsilon_{1}}$ & $\eta_{\varepsilon_{2}}$ \\
\hline 44 & 179 & -10 & 20 \\
47 & 191 & -26 & 0 \\
49 & 199 & -18 & 0 \\
52 & 211 & -6 & 12 \\
55 & 223 & -14 & 0 \\
56 & 227 & -10 & 20 \\
59 & 239 & -6 & 12 \\
62 & 251 & -14 & 28 \\
65 & 263 & -26 & 0 \\
67 & 271 & -22 & 0 \\
\hline
\end{tabular}

\begin{tabular}{|c|c|c|c|}
\hline$r$ & $p$ & $\eta_{\varepsilon_{1}}$ & $\eta_{\varepsilon_{2}}$ \\
\hline 70 & 283 & -6 & 12 \\
76 & 307 & -6 & 12 \\
77 & 311 & -38 & 0 \\
82 & 331 & -6 & 12 \\
86 & 347 & -10 & 20 \\
89 & 359 & -38 & 0 \\
91 & 367 & -18 & 0 \\
94 & 379 & -6 & 12 \\
95 & 383 & -34 & 0 \\
104 & 419 & -18 & 36 \\
\hline
\end{tabular}

\begin{tabular}{|c|c|c|c|}
\hline$r$ & $p$ & $\eta_{\varepsilon_{1}}$ & $\eta_{\varepsilon_{2}}$ \\
\hline 107 & 431 & -42 & 0 \\
109 & 439 & -30 & 0 \\
110 & 443 & -10 & 20 \\
115 & 463 & -14 & 0 \\
116 & 467 & -14 & 28 \\
119 & 479 & -50 & 0 \\
121 & 487 & -14 & 0 \\
122 & 491 & -18 & 36 \\
124 & 499 & -6 & 12 \\
125 & 503 & -42 & 0 \\
\hline
\end{tabular}

\section{Appendix: Some facts on SPIn groups AND SPIN REPRESEnTATIONS}

In this Appendix we collect some facts on conjugacy classes on $\operatorname{Spin}(n)$ and on spin representations that are used in the body of the paper. For standard facts on spin geometry we refer to [LM] or [Fr2].

We consider $(L, S)$, an irreducible complex representation of the Clifford algebra $\mathbb{C l}(n)$, restricted to $\operatorname{Spin}(n)$. The complex vector space $\mathrm{S}$ has dimension $2^{m}$ with $m=\left[\frac{n}{2}\right]$ and is called the spinor space. We have that $\mathrm{S}=\sum_{I \subset\{1, \ldots, m\}} \mathrm{S}_{\lambda_{I}}$, where $\mathrm{S}_{\lambda_{I}}$ denotes the weight space corresponding to the weight

$$
\lambda_{I}=\frac{1}{2}\left(\sum_{i=1}^{m} \varepsilon_{i}\right)-\sum_{i \in I} \varepsilon_{i}
$$

Here $\varepsilon_{j}$ is given on the Lie algebra of $T$, $\mathfrak{t}$, by $\varepsilon_{j}\left(\sum_{k=1}^{m} c_{k} e_{2 k-1} e_{2 k}\right)=2 i c_{j}$. All weights have multiplicity 1 . If $n$ is odd, then $(L, \mathrm{~S})$ is irreducible for $\operatorname{Spin}(n)$ and is called the spin representation. If $n$ is even, then the subspaces

$$
\mathrm{S}^{+}:=\sum_{|I|=\text { even }} \mathrm{S}_{\lambda_{I}}, \quad \mathrm{~S}^{-}:=\sum_{|I|=\text { odd }} \mathrm{S}_{\lambda_{I}},
$$

are $\operatorname{Spin}(n)$-invariant and irreducible of dimension $2^{m-1}$. If $L^{ \pm}$denotes the restricted action of $L$ on $\mathrm{S}^{ \pm}$, then $\left(L^{ \pm}, \mathrm{S}^{ \pm}\right)$are called the half-spin representations of $\operatorname{Spin}(n)$. We shall write $\left(L_{n}, \mathrm{~S}_{n}\right)\left(\operatorname{resp} .\left(L_{n}^{ \pm}, \mathrm{S}_{n}^{ \pm}\right)\right)$for $(L, \mathrm{~S}),\left(\operatorname{resp} .\left(L^{ \pm}, \mathrm{S}^{ \pm}\right)\right)$when we wish to specify the dimension. We have the following well-known facts:

$$
\begin{array}{ll}
\left(L_{n \mid \operatorname{Spin}(n-1)}^{ \pm}, \mathrm{S}_{n}^{ \pm}\right) \simeq\left(L_{n-1}, \mathrm{~S}_{n-1}\right) & \text { if } n \text { is even, } \\
\left(L_{n \mid \operatorname{Spin}(n-1)}, \mathrm{S}_{n}\right) \simeq\left(L_{n-1}^{+}, \mathrm{S}_{n-1}^{+}\right) \oplus\left(L_{n-1}^{-}, \mathrm{S}_{n-1}^{-}\right) & \text {if } n \text { is odd } .
\end{array}
$$
$T$.

The next lemma gives the values of the characters of $L_{n}$ and $L_{n}^{ \pm}$on elements of

Lemma 6.1. If $n=2 m$, then

$$
\chi_{L_{n}^{ \pm}}\left(x\left(t_{1}, \ldots, t_{m}\right)\right)=2^{m-1}\left(\prod_{j=1}^{m} \cos t_{j} \pm i^{m} \prod_{j=1}^{m} \sin t_{j}\right) .
$$

If $n=2 m$ or $n=2 m+1$, then

$$
\chi_{L_{n}}\left(x\left(t_{1}, \ldots, t_{m}\right)\right)=2^{m} \prod_{j=1}^{m} \cos t_{j} .
$$


Proof. Assume first that $n=2 m$ is even and proceed by induction on $m$. For $m=1$, (6.4) clearly holds. Assume it holds for $n=2 m$. Set $I_{m}=\{1, \ldots, m\}$. Now, $\chi_{L_{n+2}^{+}}\left(x\left(t_{1}, \ldots, t_{m+1}\right)\right)$ equals

$$
\begin{aligned}
& =e^{i\left(\sum_{1}^{m+1} t_{j}\right)} \sum_{\substack{I \subset I_{m+1} \\
|I| \text { even }}} e^{-2 i \sum_{j \in I} t_{j}} \\
& =e^{i t_{m+1}} e^{i\left(\sum_{1}^{m} t_{j}\right)}\left(\sum_{\substack{I \subset I_{m} \\
|I| \text { even }}} e^{-2 i \sum_{j \in I} t_{j}}+e^{-2 i t_{m+1}} \sum_{\substack{I \subset I_{m} \\
|I| \text { odd }}} e^{-2 i \sum_{j \in I} t_{j}}\right) \\
& =e^{i t_{m+1}} \chi_{L_{n}^{+}}\left(x\left(t_{1}, \ldots, t_{m}\right)\right)+e^{-i t_{m+1}} \chi_{L_{n}^{-}}\left(x\left(t_{1}, \ldots, t_{m}\right)\right) \\
& =2^{m-1}\left(e^{i t_{m+1}}\left(\prod_{j=1}^{m} \cos t_{j}+i^{m} \prod_{j=1}^{m} \sin t_{j}\right)+e^{-i t_{m+1}}\left(\prod_{j=1}^{m} \cos t_{j}-i^{m} \prod_{j=1}^{m} \sin t_{j}\right)\right) \\
& =2^{m}\left(\prod_{j=1}^{m+1} \cos t_{j}+i^{m+1} \prod_{j=1}^{m+1} \sin t_{j}\right) .
\end{aligned}
$$

The calculation for $\chi_{L_{n+2}^{-}}$is analogous. By adding both $\chi_{L_{n}^{+}}\left(x\left(t_{1}, \ldots, t_{m}\right)\right)$ and $\chi_{L_{\bar{n}}^{-}}\left(x\left(t_{1}, \ldots, t_{m}\right)\right)$ we get the asserted expression for $\chi_{L_{n}}\left(x\left(t_{1}, \ldots, t_{m}\right)\right)$ if $n=2 m$. If $n=2 m+1$, then $\chi_{L_{n}}\left(x\left(t_{1}, \ldots, t_{m}\right)\right)=\chi_{L_{n-1}}\left(x\left(t_{1}, \ldots, t_{m}\right)\right)$, hence the result follows.

The next lemma gives some useful facts on conjugacy classes of elements in $\operatorname{Spin}(n)$. We include a proof for completeness.

Lemma 6.2. Let $x, y \in \operatorname{Spin}(n-1)$ be conjugate in $\operatorname{Spin}(n)$.

(i) If $n$ is even, then $x, y$ are conjugate in $\operatorname{Spin}(n-1)$.

(ii) If $n$ is odd, then $y$ is conjugate to $x$ or to $-e_{1} x e_{1}$ in $\operatorname{Spin}(n-1)$.

Proof. If $n=2 m$ is even, then the restriction map from the representation ring $R(\operatorname{Spin}(2 m))$ to $R(\operatorname{Spin}(2 m-1))$ is onto, hence the assertion of the lemma follows.

If $n=2 m+1$, we may assume that $x=x\left(t_{1}, \ldots, t_{m}\right), y=x\left(t_{1}^{\prime}, \ldots, t_{m}^{\prime}\right)$ lie in the maximal torus $T$, where $x\left(t_{1}, \ldots, t_{m}\right)=\prod_{j=1}^{m}\left(\cos t_{j}+\sin t_{j} e_{2 j-1} e_{2 j}\right)$.

Now, if $x$ and $y$ are conjugate in $\operatorname{Spin}(2 m+1)$, then $\mu(x), \mu(y)$ are conjugate in $\mathrm{SO}(2 m+1)$, and this implies that, after reordering, we must have $t_{i}^{\prime}= \pm t_{i}$ for $1 \leq i \leq m$.

Furthermore if $1 \leq j \leq m$ we have

$$
\begin{aligned}
e_{2 j-1} e_{n} x\left(t_{1}, \ldots, t_{m}\right)\left(e_{2 j-1} e_{n}\right)^{-1} & =e_{2 j-1} x\left(t_{1}, \ldots, t_{j}, \ldots, t_{m}\right)\left(e_{2 j-1}\right)^{-1} \\
& =x\left(t_{1}, \ldots,-t_{j}, \ldots, t_{m}\right) .
\end{aligned}
$$

Hence, if $x=x\left(t_{1}, \ldots, t_{m}\right)$, then

$$
\left(e_{2 j-1} e_{2 k-1}\right) x\left(e_{2 j-1} e_{2 k-1}\right)^{-1}=x\left(t_{1}, \ldots,-t_{j}, \ldots,-t_{k}, \ldots, t_{m}\right)
$$

for $1 \leq j, k \leq m$. Thus, for fixed $t_{1}, \ldots, t_{m}$, among the elements of the form $x\left( \pm t_{1}, \ldots, \pm t_{m}\right)$ there are at most two conjugacy classes in $\operatorname{Spin}(2 m)$ represented by $x\left( \pm t_{1}, t_{2} \ldots, t_{m}\right)$ and $x\left(-t_{1}, t_{2} \ldots, t_{m}\right)=-e_{1} x\left(t_{1}, t_{2} \ldots, t_{m}\right) e_{1}$. 
Now by Lemma 6.1, we have that

$$
\chi_{L_{n-1}^{ \pm}}\left(x\left(t_{1}, \ldots, t_{m}\right)\right)=2^{m-1}\left(\prod_{j=1}^{m} \cos t_{j} \pm i^{m} \prod_{j=1}^{m} \sin t_{j}\right) .
$$

This implies that $x\left(t_{1}, t_{2} \ldots, t_{m}\right)$ and $x\left(-t_{1}, t_{2} \ldots, t_{m}\right)$ are not conjugate unless $t_{j} \in \pi \mathbb{Z}$ for some $j$. On the other hand, if this is the case, then clearly $e_{1} e_{2 j-1} \in$ $\operatorname{Spin}(n-1)$ conjugates one element into the other. This completes the proof of the lemma.

Remark 6.3. The lemma shows that generically, if $n$ is odd, $x\left(t_{1}, t_{2}, \ldots, t_{m}\right)$ and $x\left(-t_{1}, t_{2}, \ldots, t_{m}\right)$ are conjugate in $\operatorname{Spin}(n)$ but not in $\operatorname{Spin}(n-1)$.

We now consider the special case when $t_{i} \in \frac{\pi}{2} \mathbb{Z}$ for all $i$; then $\mu(x)$ has order 2 (or 1). Set $g_{h}=e_{1} e_{2} \ldots e_{2 h-1} e_{2 h} \in \operatorname{Spin}(n)$ for $1 \leq h \leq m=\left[\frac{n}{2}\right]$. Thus $g_{h}=x(\underbrace{\frac{\pi}{2}, \ldots, \frac{\pi}{2}}_{h}, 0, \ldots, 0)$ and $-g_{h}=x(\underbrace{-\frac{\pi}{2}, \frac{\pi}{2}, \ldots, \frac{\pi}{2}}_{h}, 0, \ldots, 0)$.

Corollary 6.4. If $h<m$, then $g_{h}$ and $-g_{h}$ are conjugate in Spin $(n-1)$. If $h=m$ and $n=2 m$, then $\chi_{L_{n}^{ \pm}}\left(g_{m}\right)= \pm 2^{m-1} i^{m}$, hence $g_{m}$ and $-g_{m}$ are not conjugate. If $h=m$ and $n=2 m+1$, then $\chi_{L_{n-1}^{+}}\left( \pm g_{m}\right)= \pm 2^{m-1} i^{m}$, hence $g_{m}$ and $-g_{m}$ are conjugate in Spin $(n)$ but not in Spin $(n-1)$.

Proof. The first assertion in the corollary follows immediately from the proof of Lemma 6.2. The remaining assertions are clear in light of Lemma 6.1 Indeed, for $h<m$ we have $e_{1} e_{n} g_{h}\left(e_{1} e_{n}\right)^{-1}=-g_{h}$.

Recall that for any $u \in \mathbb{R}^{n} \backslash\{0\}$, left Clifford multiplication by $u$ on $\mathrm{S}$ is given by $u \cdot w=L(u)(w)$ for $w \in \mathrm{S}$. We fix an inner product $\langle$,$\rangle on \mathrm{S}$ such that $L(u)$ is skew Hermitian, hence $\langle$,$\rangle is \operatorname{Spin}(n)$-invariant. Note that $L(u)^{2}=-\|u\|^{2} I d$. Hence, $\mathrm{S}$ decomposes $\mathrm{S}=\mathrm{S}_{u}^{+} \oplus \mathrm{S}_{u}^{-}$, where $\mathrm{S}_{u}^{ \pm}$denotes the eigenspaces, of dimension $2^{m-1}$, of $L(u)$ with eigenvalues $\mp i\|u\|$.

Definition 6.5. If $u \in \mathbb{R}^{n} \backslash\{0\}$ set

$$
\operatorname{Spin}(n-1, u):=\left\{g \in \operatorname{Spin}(n): g u g^{-1}=u\right\} .
$$

Clearly $\operatorname{Spin}\left(n-1, e_{n}\right)=\operatorname{Spin}(n-1)$, and for general $u$, if $h_{u} \in \operatorname{Spin}(n)$ is such that $h_{u} u h_{u}{ }^{-1}=\|u\| e_{n}$, then $h_{u} \operatorname{Spin}(n-1, u) h_{u}^{-1}=\operatorname{Spin}(n-1)$. We note that for any $g \in \operatorname{Spin}(n-1, u), L(g)$ commutes with $L(u)$, hence $L(g)$ preserves the eigenspaces $\mathrm{S}_{u}^{ \pm}$.

The following lemma is used in the proof of Theorem 2.5

Lemma 6.6. Let $\operatorname{Spin}(n-1, u)$ be as in (6.6). Then as $\operatorname{Spin}(n-1)$-modules: $S_{e_{n}}^{ \pm} \simeq$ $\left(L_{n-1}^{ \pm}, S_{n-1}^{ \pm}\right)$if $n$ is odd and $S_{e_{n}}^{ \pm} \simeq\left(L_{n-1}, S_{n-1}\right)$ if $n$ is even. As $\operatorname{Spin}(n-1, u)$ modules we have that $S_{u}^{ \pm}=L\left(h_{u}\right) S_{n-1}^{ \pm}$if $n$ is odd and $S_{u}^{ \pm}=L\left(h_{u}\right) S_{n-1}$ if $n$ is even, with action given by $L\left(h_{u} x h_{u}^{-1}\right)=L\left(h_{u}\right) L(x) L\left(h_{u}^{-1}\right)$ for any $x \in \operatorname{Spin}(n-1)$.

Proof. $L\left(e_{n}\right)$ commutes with the action of $\operatorname{Spin}(n-1)$ on $\mathrm{S}_{n}$ and, on the other hand, $\mathrm{S}_{n}=\mathrm{S}_{n-1}^{+} \oplus \mathrm{S}_{n-1}^{-}$as a $\operatorname{Spin}(n-1)$-module.

If $n$ is odd, then $S_{n-1}^{ \pm}$are inequivalent representations of $\operatorname{Spin}(n-1)$, hence $L\left(e_{n}\right) \mathrm{S}_{n-1}^{ \pm}=\mathrm{S}_{n-1}^{ \pm}$and by Schur's lemma, $L\left(e_{n}\right)$ must act by multiplication by a scalar on each of them. By using the explicit description of $L$ in [Kn, pp. 286-288, one verifies that $L\left(e_{n}\right)$ acts by $\mp i$ on $S_{n-1}^{ \pm}$, that is, $S_{e_{n}}^{ \pm} \simeq \mathrm{S}_{n-1}^{ \pm}$. 
If $n$ is even, then $\mathrm{S}_{n}^{ \pm}$both restrict to $\mathrm{S}_{n-1}$ as $\operatorname{Spin}(n-1)$-modules. Since the $\pm i$-eigenspaces of $L\left(e_{n}\right)$ are stable by $\operatorname{Spin}(n-1)$, they must both be equivalent to $\mathrm{S}_{n-1}$.

The remaining assertions are easily verified.

\section{REFERENCES}

[AB] Ammann, B., Bär C., The Dirac operator on nilmanifolds and collapsing circle bundles, Annals of Global Analysis and Geometry, 16 (221-253), 1998. MR1626659 (99h:58194)

[Ap] Apostol, T., Introduction to analytic number theory, Springer Verlag, NY, 1998. MR0434929 (55:7892)

[APS] Atiyah, M. F., Patodi V. K., Singer, I. M., Spectral asymmetry and Riemannian geometry I, II, III, Math. Proc. Cambridge Philos. Soc. 77 (43-69), 1975; 78 (405-432), 1975; 79 (71-99), 1976.

[Ba] Bär, C., Dependence of the Dirac spectrum on the spin structure, Global Analysis and Harmonic Analysis (Marseille-Luminy 1999), Sémin. \& Cong. 4, Soc. Math. Fce., (17-33), Paris 2000. MR1822353 (2002f:53080)

[BDM] Barberis, M. L., Dotti, I. G., Miatello, R. J., Clifford structures on certain locally homogeneous manifolds. Annals Global Analysis and Geometry 13, (289-301), 1995. MR.1344484 (96g:53060)

[BGR] Bovdi, V. A., Gudivok, P. M., Rudko, V. P., Torsion-free groups with indecomposable holonomy group. I. J. Group Theory 5, (75-96), 2002. MR.1879518 (2002k:20090)

[BGV] Berline, N., Getzler E., Vergne M., Heat kernels and Dirac operators, Springer-Verlag, GMW 298, 1992. MR1215720(94e:58130)

[Ch] Charlap, L., Bieberbach groups and flat manifolds, Springer-Verlag, Universitext, 1988. MR0862114(88j:57042)

[DM] Dotti, I. G., Miatello, R. J., Isospectral compact flat manifolds, Duke Math. J. 68 (489498), 1992. MR1194952 (94b:53066)

[Fr] Friedrich, T., Die Abhängigkeit des Dirac-Operators von der Spin-Struktur, Coll. Math. XLVII (57-62), 1984. MR0750754 (85h:58175)

[Fr2] Friedrich, T., Dirac operator in Riemannian geometry, Amer. Math. Soc. GSM 25, 1997.

[Gi] Gilkey, P. B., The residue of the global $\eta$-function at the origin, Advances in Math. 40, (290-307), 1981. MR0624667 (83c:58075)

[HZ] Hirzebruch, F., Zagier D., The Atiyah-Singer Theorem and Elementary Number Theory, Publish or Perish, Math. Lecture Series 3, 1974. MR0650832 (58:31291)

[IK] Im, S. M., Kim, H. K., Compact flat manifolds with non-vanishing Stiefel-Whitney classes, Topology and its Applications 96, (267-276), 1999. MR1709693 (2001i:57037)

[JR] Johnson, F. E. A., Rees, E. G., Kähler groups and rigidity phenomena, Math. Proc. Cambridge Philos. Soc. 109, (31-44), 1991. MR1075119 (91i:58040)

[KL] Krasikov, I., Litsyn, S., On integral zeros of Krawtchouk polynomials, J. Combin. Theory A 74 (71-99), 1996. MR.1383506 (97i:33005)

[Kn] Knapp, A. W., Lie groups: Beyond an introduction, Birkhäuser, Progress in Math. 140, 1996. MR $1399083(98 \mathrm{~b}: 22002)$

[LM] Lawson, H. B., Michelsohn, M. L., Spin geometry, Princeton University Press, NJ, 1989. MR.1031992 (91g:53001)

[LS] Lee, R., Szczarba R. H., On the integral Pontrjagin classes of a Riemannian flat manifold, Geom. Dedicata 3 (1-9), 1974. MR0341341 (49:6092)

[MP] Miatello, R. J., Podestá, R. A., Spin structures and spectra of $\mathbb{Z}_{2}^{k}$-manifolds, Math. Zeitschrift 247 (319-335), 2004. MR2064055

[MPR] Miatello, R. J., Podestá, R. A., Rossetti, J. P., $\mathbb{Z}_{2}^{k}$-manifolds are isopectral on forms, preprint 2004.

[MR] Miatello, R. J., Rossetti, J. P., Isospectral Hantzsche-Wendt manifolds, J. Reine Angew. Math. 515 (1-23), 1999. MR.1717633 (2000k:57037)

[MR2] Miatello, R. J., Rossetti, J. P., Hantzsche-Wendt manifolds of dimension 7, Diff. Geom. Appl., Proceedings of the $7^{\text {th }}$ International Conference, Masaryk Univ., Brno (379-391), 1999. MR1708926 (2001c:58032)

[MR3] Miatello, R. J., Rossetti, J. P., Flat manifolds isospectral on p-forms, Jour. Geom. Anal. 11 (647-665), 2001. MR1861302 (2003d:58053) 
[MR4] Miatello, R. J., Rossetti, J. P., Comparison of twisted Laplace p-spectra for flat manifolds with diagonal holonomy, Ann. Global Anal. Geom. 21 (341-376), 2002. MR.1910457 (2003f:58065)

[MR5] Miatello, R. J., Rossetti, J. P., Length spectra and P-spectra of compact flat manifolds, Jour. Geom. Anal. 13, 4, (631-657), 2003. MR2005157 (2005a:58053)

[Pf] Pfäffle, F., The Dirac spectrum of Bieberbach manifolds, J. Geom. Phys. 35 (367-385), 2000. MR,1780760 (2002b:58045)

[RS] Rossetti, J. P., Szczepanski, A., Generalized Hantzsche-Wendt manifolds, Rev. Matemática Iberoamericana 21, 3, 2005, 1053-1070, arXiv:mathGT/0208205.

[SS] Sadowski, M., Szczepanski, A., Flat manifolds, harmonic spinors and eta invariants, preprint, arxiv.org/abs/math/0310183.

[Va] Vasquez, A., Flat Riemannian manifolds, J. Diff. Geometry 4 (367-382), 1970. MR0267487 (42:2389)

[Wo] Wolf, J., Spaces of constant curvature, Mc Graw-Hill, NY, 1967. MR.0217740 (36:829)

[WW] Whittaker, E. T., Watson, G. N., A course of modern analysis, Cambridge Univ. Press, London, 1963. MR1424469 (97k:01072)

FaMAF-Ciem, Universidad Nacional de Córdoba, Argentina

E-mail address: miatello@mate.uncor.edu

FamaF-Ciem, Universidad Nacional de Córdoba, Argentina

E-mail address: podesta@mate.uncor.edu 